\title{
A Two-Stage Decomposition of High School Timetabling applied to cases in Denmark
}

\section{Sørensen, Matias; Dahms, Florian H.W.}

Published in:

Computers and Operations Research

Link to article, DOI:

10.1016/j.cor.2013.08.025

Publication date:

2014

Link back to DTU Orbit

Citation (APA):

Sørensen, M., \& Dahms, F. H. W. (2014). A Two-Stage Decomposition of High School Timetabling applied to cases in Denmark. Computers and Operations Research, 43, 36-49. https://doi.org/10.1016/j.cor.2013.08.025

\section{General rights}

Copyright and moral rights for the publications made accessible in the public portal are retained by the authors and/or other copyright owners and it is a condition of accessing publications that users recognise and abide by the legal requirements associated with these rights.

- Users may download and print one copy of any publication from the public portal for the purpose of private study or research.

- You may not further distribute the material or use it for any profit-making activity or commercial gain

- You may freely distribute the URL identifying the publication in the public portal

If you believe that this document breaches copyright please contact us providing details, and we will remove access to the work immediately and investigate your claim. 


\title{
A Two-Stage Decomposition of High School Timetabling applied to cases in Denmark
}

\author{
Matias Sørensen $^{\mathrm{a}, \mathrm{b}, *}$, Florian H.W. Dahms ${ }^{\mathrm{c}}$ \\ ${ }^{a}$ Section of Operations Research, Department of Management Engineering, Technical University of Denmark, DK-2800 Kgs. Lyngby, Denmark \\ ${ }^{b}$ MaCom A/S, Vesterbrogade 48, 1., DK-1620 Copenhagen V, Denmark \\ ${ }^{c}$ Chair of Operations Research, RWTH Aachen University, Kackertstraße 7, 52072 Aachen, Germany
}

\begin{abstract}
Integer Programming (IP) has been used to model educational timetabling problems since the very early days of Operations Research. It is well recognized that these IP models in general are hard to solve, and this area of research is dominated by heuristic solution approaches. In this paper a Two-Stage Decomposition of an IP model for a practical case of high school timetabling is shown. This particular timetabling problem consists of assigning lectures to both a timeslot and a classroom, which is modeled using a very large amount of binary variables. The decomposition splits this model into two separate problems (Stage I and Stage II) with far less variables. These two separate problems are solved in sequence, such that the solution for the Stage I model is given as input to the Stage II model, implying that irreversible decisions are made in Stage I. However, the objective of the Stage II model is partly incorporated in the Stage I model by exploiting that Stage II can be seen as a minimum weight maximum matching problem in a bipartite graph. This theoretically strengthens the decomposition in terms of global optimality. The approach relies on Hall's theorem for the existence of matchings in bipartite graphs, which in its basic form yields an exponential amount of constraints in the Stage I model. However, it is shown that only a small subset of these constraints is needed, making the decomposition tractable in practice for IP solvers. To evaluate the decomposition, 100 real-life problem instances from the database of the high school ERP system Lectio are used. Computational results show that the decomposition performs significantly better than solving the original IP, in terms of both found solutions and bounds.
\end{abstract}

Keywords: High School Timetabling, Integer Programming, Decomposition, Bipartite Matching

\section{Introduction}

Integer Programming (IP) has been used to model educational timetabling problems since the very early days of Operations Research (see e.g. Gotlieb (1962) and Lawrie (1969)). It is well recognized that these IP models in general are hard to solve (most forms of educational timetabling are in fact $\mathcal{N} \mathcal{P}$-hard (Bardadym (1996))), and this area of research is dominated by heuristic solution approaches.

In this paper a large IP model for a real-world case of high school timetabling is considered, which has previously been shown to be a challenge for state-of-the-art MIP solvers. We consider a basic version of this IP, which includes the essential constraints of most timetabling problems. An innovative decomposition of this model is shown, which proves to be more efficient to solve.

When facing a hard IP model, decomposition is a commonly used tool to help speed up the solution procedure. Perhaps the most successful decomposition method in recent years is Column Generation (CG). However, not many papers on CG and timetabling models are found in the literature, and it seems that only relatively small instances have been attempted. Papoutsis et al. (2003) uses CG to solve a Greek case of high school timetabling, with the largest instance containing 9 class section, 21 teachers and 306 teaching hours. Santos et al. (2012) handle larger instances,

${ }^{*}$ Corresponding author

Email address: msso@dtu.dk (Matias Sørensen) 
but only generate lower bounds. Qualizza and Serafini (2005) describe a CG procedure for a university timetabling problem with 63 courses and 25 timeslots. The real-world instances considered in this paper are of much larger size.

A crucial part of a CG procedure is the identification of a block-diagonal structure in the problem, otherwise the $\mathrm{CG}$ procedure is most likely not efficient. For the high school timetabling problem described in this paper, it has not been possible to identify such a structure. Therefore this paper shows a different type of decomposition, a Two-Stage Decomposition (TSD). Such an approach was first used for timetabling applications in Lach and Lübbecke (2008) and Lach and Lübbecke (2012) with great success for the curriculum-based university course timetabling problem. The goal of this paper will be to modify the aforementioned approach to be applicable for the high school timetabling problem - giving special attention to the high school system in Denmark.

The considered timetabling problem essentially consists of assigning lectures to rooms and timeslots, which is commonly modeled using a very large amount of binary variables. There are three key points to the TSD:

- By substitution, the total amount of variables is significantly reduced, while linearity is maintained.

- Instead of solving the entire model at once, it can be solved in a two-stage fashion. I.e. both the set of variables and constraints are divided into two distinct sets, corresponding to two smaller IPs (denoted Stage I and Stage II, respectively).

- It will be evident that, except for two soft-constraints, this decomposition maintains optimality of the original model.

The outline of the TSD is to first solve Stage I, which provides a solution where lectures are assigned to timetslots. This partial solution is given as input to Stage II, which will assign rooms to the lectures, obtaining a solution for the original problem. The drawback of this decomposition is that the timeslots assigned to lectures in Stage I are considered as fixed by the Stage II model, which might prevent an optimal allocation of rooms to lectures. However, by exploiting the structure of the Stage II model, the Stage I model can be constrained in such a way that some penalties for assigning rooms to lectures are handled implicitly. Note that if all penalties for room assigning could be handled implicitly, the approach would be exact. However, two soft-constraints are not fully incorporated, so only a lower bound on the room penalties are known by the Stage I model. In fact, one of these soft-constraints are not handled at all by the described approach. Despite this, it seems likely that incorporating this lower bound in the Stage I model will provide better results overall (assuming that computing the lower bound does not have very bad influence on the computational efforts of the used IP solver). I.e. instead of the Stage I model being completely unaware of the penalties for room allocation, it seems better to at least incorporate some of them. Furthermore, the decomposition of the problem into two smaller problems presents a big advantage in terms of reduction in the number of variables. Therefore the overall benefits of the TSD out-weight the downsides, and computational results will show that it is indeed way more effective than solving the original IP.

The contributions of this paper are the following: 1) It is shown that the approach from Lach and Lübbecke (2008) can also be applied to a high school timetabling problem originating from a practical setting, and by extensive computational results it is argued that the TSD is more effective than solving the original IP. Notice that a similar composition is briefly mentioned in Sørensen and Stidsen (2013) for the same high school timetabling problem, but this paper enhances the approach such that the theoretical maximum gap from optimality is narrowed. The presented approach turns out to be the most efficient exact algorithm for the problem so far. 2) Generally, it is shown how this type of decomposition can be applied to models with set-packing structure, by modifying the underlying equations originating from Hall's Theorem for matchings in bipartite graphs. 3) It is shown how the room-priorities of lectures can be handled, by adding a lower bound on the corresponding penalties to the Stage I model. This facilitates the quality of the solutions found, as shown by the computational results.

We expect that the basic structure required for applying the TSD can be found in other timetabling problems as well, and therefore the decomposition can potentially be used more broadly than the case of high school timetabling shown in this paper. This seems likely because the essential constraints used in the decomposition are among the most common ones found in timetabling problems.

The paper is structured as follows. First related papers are described in Section 2. The basic IP model is introduced in Section 3, including the essential constraints. Section 4 shows the TSD of this model, and derives the lower bound on room allocation penalties for the Stage I model. Section 5 extends the model so it encapsulates a practical version 
of the high school timetabling problem, defined by the online high school administration system Lectio. Section 6 shows computational results, comparing the decomposition to previous approaches for 100 problem instances taken from the Lectio database. Section 7 concludes on our findings.

\section{Related work}

Integer Programming has been used to model various educational timetabling problems. However, heuristics are still the most popular method for these problems, see surveys Schaerf (1999) and Pillay (2013). In terms of IP, de Werra (1985) describes what is called 'a simple model' for the class-teacher problem, and existence of solutions is proven under certain circumstances using graph theoretical models. The problems considered are feasibility problems, and soft constraints are not added to the models. Birbas et al. (1997) describes a 'fully defined' IP model for Greek secondary schools, which is evaluated on five different schools with success. Avella et al. (2007) formulates an IP model which is used to solve small instances of various origin. The IP is solved within a VLSN algorithm, with good results.

For the related university course timetabling problem, Daskalaki et al. (2004) presents a model which schedules courses to timeslots and classrooms, using many so called operational rules. Three different problem instances of significant size are all solved to optimality using CPLEX. MirHassani (2006) describes the problem for an Iranian university, and reports good results by applying the XA solver. In Dimopoulou and Miliotis (2001) an IP model is used to solve the timetabling problem for The Athens University of Economics and Business.

Decomposition of IP models for educational timetabling is not a very well researched topic. Burke et al. (2010) state that: In the timetabling community, the "times first, rooms second" decomposition is a standard procedure. However, it seems that this procedure has not been applied much in context of IP models. Burke and Newall (1999) apply the procedure in context of an Evolutionary Algorithm for Examination Timetabling. In terms of multistagedecompositions, the importance of Lach and Lübbecke (2008) and Lach and Lübbecke (2012) has already been discussed. Carter (1983) presents an interesting decomposition algorithm for course timetabling with elective courses. Stating the problem in terms of a vertex coloring problem facilitates the decomposition of the graph by cliques, such that the subproblem defined by each clique is solved separately.

In Burke et al. (2010), experiments are conducted on disabling different combinations of soft-constraint penalties of the Udine Course Timetabling Problem, including one where all room penalties on room allocation are disabled. Thereby a similar decomposition to that of Lach and Lübbecke (2012) is obtained.

Daskalaki and Birbas (2005) presents an approach for university timetabling, where courses are first assigned to days (skipping some requirements for compactness), and in the following stage the timetable for each day is treated locally (enforcing the compactness). Convincing computational results are shown. In Birbas et al. (2009), a high school timetabling problem is solved by first allocating 'work shifts' to teachers, and then solving the actual timetabling problem. This is related to the type of decomposition performed in this paper. Badri (1996) uses a related approach for university course timetabling, where faculties are first assigned to courses, and then faculties are assigned to timeslots. However the problems solved are tiny.

Recently, high school timetabling received attention in the International Timetabling Competition 2011 (ITC2011), see Post et al. (2012a). This competition built upon an uniform format for formulating problem instances (and their solutions), known as XHSTT (Post et al. (2012b)). Currently, around 50 problem instances are available in this format. The problem considered in this paper deviates from the XHSTT format in several important ways, which is beyond the scope of this section to elaborate on. Even though many researches participated in ITC2011, it seems that all were applying heuristics.

\section{An Integer Programming Model for High School Timetabling}

As the origin for our approach lies the IP model presented in Sørensen and Stidsen (2013). To make a clear presentation of the TSD, this IP model is reduced to its essential parts, which is described in the following. In Section 5, the full IP model is shown in context of the TSD.

A set of events $\mathcal{E}$ is given. Each event generally represents one lecture, which is defined as a meeting between specific resources, with a certain subject as teaching-objective. The set of resources is denoted $\mathcal{A}$. The goal of the high 
school timetabling problem is to assign each event to a room and to a timeslot, such that no conflicts among resources occur. The set of rooms and timeslots are denoted $\mathcal{R}$ and $\mathcal{T}$, respectively. The decision variable $x_{e, r, t} \in\{0,1\}$ takes value 1 if event $e \in \mathcal{E}$ is assigned room $r \in \mathcal{R}$ and timeslot $t \in \mathcal{T}$. To ensure a feasible solution exists, both the set of timeslots $\mathcal{T}$ and the set of rooms $\mathcal{R}$ are extended with a single dummy element, i.e. $\mathcal{T}=\left\{\mathcal{T} \cup t_{D}\right\}$ and $\mathcal{R}=\left\{\mathcal{R} \cup r_{D}\right\}$. This should be interpreted in the way that assigning to these dummy-elements actually means that no timeslot/room was assigned to the event. Thereby the goal of the IP is to assign as many events as possible to a timeslot and/or a room. From a practical point of view this is desirable, as the model is used in a decision support context where it might not be evident how to handle infeasibility. $\phi_{e, t} \in \mathbb{R}^{+}$denotes the penalty for assigning event $e \in \mathcal{E}$ to timeslot $t \in \mathcal{T}$, and $\pi_{e, r} \in \mathbb{R}^{+}$denotes the penalty for assigning event $e \in \mathcal{E}$ to room $r \in \mathcal{R}$. Major penalties are given for assignments to the dummy-elements, i.e.

$$
\begin{array}{ll}
\phi_{e, t_{D}} \gg \phi_{e, t} \quad \forall e \in \mathcal{E}, t \in \mathcal{T} \backslash t_{D} \\
\pi_{e, r_{D}} \gg \pi_{e, r} \quad \forall e \in \mathcal{E}, r \in \mathcal{R} \backslash r_{D}
\end{array}
$$

A room might be unavailable in certain timeslots, indicated by the binary parameter $G_{r, t} \in\{0,1\}$, which takes value 1 if room $r \in \mathcal{R}$ is available in timeslot $t \in \mathcal{T}$, and 0 otherwise. Furthermore, a set of eligible rooms exists for each event. Let parameter $K_{e, r} \in\{0,1\}$ take value 1 if event $e \in \mathcal{E}$ can take place in room $r \in \mathcal{R}$, and 0 otherwise. Each event requires a fixed set of resources. Let $E_{a}^{\prime}, a \in \mathcal{A}$, denote the set of events where resource $a$ participates.

We include in the model a set of constraints which will be described later, denoted by the constraint-set $P_{\text {other }}$, slightly abusing notation. These constraints define various other important criteria, such as forbidden timeslots for certain events, events which must be placed in the same timeslots, etc. Since these constraints are not required for describing the decomposition, their definitions are postponed to Section 5. We allow the set of constraints $P_{\text {other }}$ to also denote soft-constraints (i.e. constraints which result in a weighted penalty in the objective function if it is not fulfilled). Thereby these constraints contain all necessary for conditions for modeling the timetabling instances in question, and represents a large set of distinct types of constraints. It will be argued in the next section that these constraints can be handled in the decomposition such that optimality of the IP model is not lost, with one exception.

Model (3) shows the IP model.

IP Model for High School Timetabling

$$
\begin{aligned}
& \min \quad w=\sum_{e \in \mathcal{E}, r \in \mathcal{R}, t \in \mathcal{T}}\left(\phi_{e, t}+\pi_{e, r}\right) x_{e, r, t} \\
& \text { s.t. } \\
& \text { (one time/room) } \sum_{r \in \mathcal{R}, t \in \mathcal{T}} x_{e, r, t}=1 \quad \forall e \in \mathcal{E} \\
& \text { (resource conf.) } \sum_{r \in \mathcal{R}, e \in E_{a}^{\prime}} x_{e, r, t} \leq 1 \quad \forall a \in \mathcal{A}, t \in \mathcal{T} \backslash t_{D} \\
& \text { (room conf.) } \sum_{e \in \mathcal{E}} x_{e, r, t} \leq G_{r, t} \forall r \in \mathcal{R} \backslash r_{D}, t \in \mathcal{T} \backslash t_{D} \\
& \text { (eligible rooms) } \sum_{t \in \mathcal{T}} x_{e, r, t} \leq K_{e, r} \forall e \in \mathcal{E}, r \in \mathcal{R} \\
& x_{e, r, t} \in P_{\mathrm{other}} \\
& x_{e, r, t} \in\{0,1\}
\end{aligned}
$$

The objective of the model is to minimize the overall penalty for assignments, given by (3a). Constraint (3b) specifies that each event must be assigned exactly one timeslot and one room. Events which require the same resource cannot be scheduled simultaneously (except in the dummy-timeslot), which is ensured by constraint ( $3 \mathrm{c}$ ). A room cannot be used by more than one event in each timeslot. This is specified in constraint (3d). The requirement for eligible rooms is specified in constraint (3e). Constraint (3f) specifies that constraints $P_{\text {other }}$ should be respected.

Theorem 3.1. The High School Timetabling Problem as specified in (3) is $\mathcal{N} \mathcal{P}$-hard.

Proof. We conduct a reduction from Vertex Coloring. Let $G=(V, E)$ be an arbitrary graph and $k$ be an arbitrary number. The question of the coloring problem would now be whether it is possible to color $G$ with $k$ colors, such that no two adjacent vertices share the same color. 
Now construct a High School Timetabling instance in the following way:

- Let there be an event for every vertex, i.e. $\mathcal{E}=V$.

- Make sure there are enough rooms for all events, therefore create a room for every event (i.e. $|\mathcal{R}|=|\mathcal{E}|$ ) and make sure all events fit in all rooms (i.e. $K_{e, r}=1 \forall e \in \mathcal{E}, r \in \mathcal{R}$ ), and that all rooms are available in all timeslots (i.e. $\left.G_{r, t}=1 \forall e \in \mathcal{E}, r \in \mathcal{R}\right)$.

- For every edge $\left\{v_{1}, v_{2}\right\} \in E$ we create a resource in $\mathcal{A}$ (i.e. $\mathcal{A}=E$ ). The events using this resource will be the vertices connected by the edge (i.e. $E_{\left\{v_{1}, v_{2}\right\}}^{\prime}=\left\{v_{1}, v_{2}\right\}$ ).

- We use exactly $k$ timeslots (i.e. $\mathcal{T}=\{1, \ldots, k\}$ ).

- The additional constraints $P_{\text {other }}$ can be dropped without loss of generality, as we impose no other restrictions on the timetabling instance. Also as we only search for a feasible solution, the soft constraints can easily be ignored.

Now we have a direct relation between the Vertex Coloring problem and the new timetabling instance. A solution of one problem can be transformed into a solution of the other by translating the colors of the vertices into timeslots, and vice versa. The rooms pose no restriction as every event can be scheduled in its own room.

Therefore solving the timetabling instance would result in solving the Vertex Coloring Problem, and the High School Timetabling problem is $\mathcal{N} \mathcal{P}$-hard.

Here we remark that Model (3) encapsulates many of the basic constraints required by most timetabling problems. If we for instance consider the XHSTT format, the basic requirement is to assign events to timeslots and resources (corresponding to rooms in our cases), subject to no clashes between resources. Therefore it is believed that the type of decomposition considered in this paper can in principle be applied to other timetabling problems as well.

\section{Two-Stage Decomposition of the Integer Programming model}

The TSD of Model (3) is performed as follows. The model is split into two stages; In Stage I, events are assigned to timeslots, and in Stage II, events are assigned to rooms. The respective decision variables for these stages are the following; $y_{e, t} \in\{0,1\}$ takes value 1 if event $e \in \mathcal{E}$ is assigned timeslot $t \in \mathcal{T}$, and 0 otherwise; $z_{e, r} \in\{0,1\}$ takes value 1 if event $e \in \mathcal{E}$ is assigned room $r \in \mathcal{R}$, and 0 otherwise. This means that constraints (3b) and (3c) are part of Stage I, and constraints (3d) and (3e) are part of Stage II.

As for constraints (3f), defined by the set $P_{\text {other }}$, it is assumed that each of the constraints in $P_{\text {other }}$ is either only touching the assignment of events to timeslots (denoted $P_{\text {timeslot }}$ ) or the assignment of events to rooms (denoted $\left.P_{\text {room }}\right)$ It is shown in Section 5 that this assumption holds, with one exception. This means that constraints $P_{\text {timeslot }}$ can be fully stated in terms of variable $y_{e, t}$, and constraints $P_{\text {room }}$ can be fully stated in terms of variable $z_{e, r}$. As constraints $P_{\text {timeslot }}$ are part of Stage I, these are handled optimally. This is different for $P_{\text {room }}$, as those constraints are harder to consider during Stage I. We will therefore address them with greater care in the next sections and show how we can add weighted room allocations to the decomposed model as a good approximation.

The solution obtained from the Stage I model is given as a parameter to the Stage II model, denoted $y_{e, t}^{*}$. The advantage of this approach is the huge reduction in the number of variables in both stages, which results in a significantly decreased solving time. The following substitution of variables are made:

$$
\begin{aligned}
& \sum_{r \in \mathcal{R}} x_{e, r, t}=y_{e, t} \quad \text { (Stage I) } \\
& x_{e, r, t}=y_{e, t}^{*} z_{e, r} \quad(\text { Stage II })
\end{aligned}
$$

The objective (3a) of the original model defines a natural objective for both Stage I and Stage II, since it can be split into two independent expressions (denoted $w^{I}$ and $w^{I I}$, respectively). If this was not the case (e.g. if an event had different priorities for rooms depending on the timeslot it was assigned), it would complicate matters in terms of the Stage I model. 
To sum up, Models (6) and (7) show Stage I and Stage II, respectively.

Stage I

$\min w^{I}=\sum_{e \in \mathcal{E}, t \in \mathcal{T}} \phi_{e, t} y_{e, t}$

s.t.

$$
\begin{array}{cl}
\text { (one timeslot) } & \sum_{t \in \mathcal{T}} y_{e, t}=1 \forall e \in \mathcal{E} \\
\text { (resource conf.) } & \sum_{e \in E_{a}^{\prime}} y_{e, t} \leq 1 \forall a \in \mathcal{A}, t \in \mathcal{T} \backslash t_{D} \\
& y_{e, t} \in P_{\text {timeslot }} \\
& y_{e, t} \in\{0,1\}
\end{array}
$$

Stage II (solution from Stage I is denoted $y_{e, t}^{*}$ )

$$
\begin{array}{ll}
\min \quad w^{I I}=\sum_{e \in \mathcal{E}, r \in \mathcal{R}} \pi_{e, r} z_{e, r} & \\
\text { s.t. } & \sum_{r \in \mathcal{R}} z_{e, r}=1 \quad \forall e \in \mathcal{E} \\
\text { (one room) } & \sum_{e \in \mathcal{E}} y_{e, t}^{*} z_{e, r} \leq G_{r, t} \forall r \in \mathcal{R} \backslash r_{D}, t \in \mathcal{T} \backslash t_{D} \\
\text { (room conf.) } \quad \leq K_{e, r} \forall e \in \mathcal{E}, r \in \mathcal{R} \\
\text { (eligible rooms) } & z_{e, r} \quad P_{e, r} \in P_{\text {room }} \\
& z_{e, r} \in\{0,1\}
\end{array}
$$

The outline of the TSD is shown in Figure 1. For a variable $x$ the star-suffixed version $x^{*}$ denotes a feasible solution. Stage I is solved using a MIP solver to obtain a solution $y_{e, t}^{*}$, which is given as input to the Stage II model. Note that Stage I possesses the coloring structure from theorem 3.1. Therefore Stage I is already a hard problem in its most basic form. Furthermore the value of the LP-relaxation of the Stage I model (denoted $w_{L P}^{I *}$ ) is a lower bound on the original model, as the Stage I model can be seen as a relaxation. Solving the Stage II model subject to the solution of the Stage I model obtains a solution $z_{e, t}^{*}$, and a solution to the original model $x_{e, r, t}^{*}$ can then be derived by equations (4) and (5).

For this paper we will solve Stage II using the specified IP even though we have not established its complexity yet. But as constructing a polynomial time algorithm for Stage II that can cope with all the additional constraints would be out of this papers scope we will postpone this to a potentially later point in time. In the computational results section we will see that solving Stage II will not be the time-wise bottleneck anyhow.

As previously discussed, the penalties for room allocation can be implicitly handled in Stage I, which is described in Section 4.1. This extension of Stage I will not only allow better solutions to be found, but possibly also improvements in the bounds found by means of the LP relaxation.

As an alternative approach, we remark that an iterative procedure could in principle be used, such that the solution obtained from the Stage II model is given as input to the Stage I model, and the whole procedure is repeated. It is however unclear how the input from the Stage II model should effect the Stage I model to obtain convergence towards better solutions in terms of the overall objective. Furthermore, such an approach would require that both Stage I and Stage II can be solved 'quickly' (for the practical problem treated in this paper, computational results will show that this is in fact not the case for the Stage I model).

\subsection{Extending Stage I with room allocation}

The key idea behind extending Stage I with room allocation penalties is to consider Stage II as a matching problem in a bipartite graph. Constraints (7e) are set aside in the following, as they have not been defined yet. However, it will 


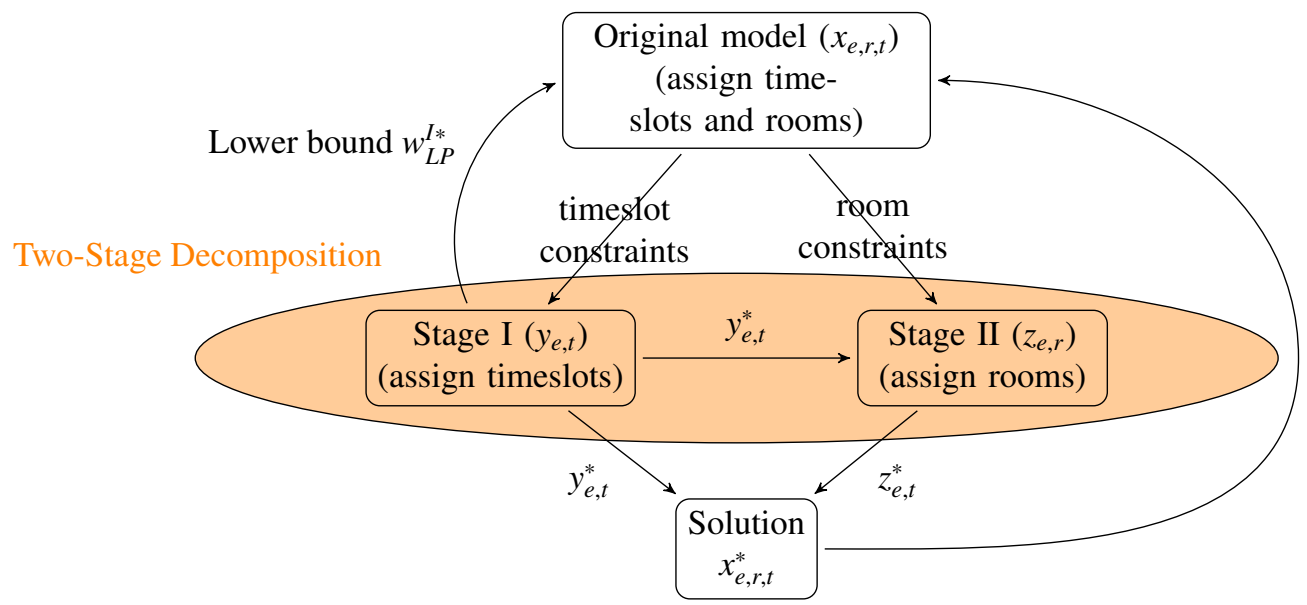

Figure 1: Two-Stage Decomposition flow chart.

be seen later that these constraints does not fully obey the matching problem structure, and therefore Stage II must be solved with a MIP solver. This means that the room penalties are only partly incorporated in Stage I, but still this seems better than having Stage I being totally unaware of these penalties, as already discussed in Section 1.

Some basic graph notation is introduced in the following. A graph is bipartite if its set of vertices can be partitioned into two sets $A$ and $B$, such that every edge has one endpoint in $A$, and the other endpoint in $B$. A matching in a graph is a set of edges such that no two of these edges share endpoints. A maximum matching is a matching that contains the largest possible number of edges. The matching number $v(G)$ of graph $G$ is the number of edges in a maximum matching. For a graph with edge-weights, a minimum weighted maximum matching is a maximum matching where the sum of the weights on the edges of the matching is minimal.

In the Stage II model (7), notice first that the only constraint which treats timeslots is constraint (7c). Since this constraint applies to timeslots individually, Model (7) can be split into $|\mathcal{T}|$ independent optimization problems. Second, assume that the minimum weighted maximum matching problem of the weighted bipartite graph $G_{t}=\left(\mathcal{E} \cup \overline{\mathcal{R}}, E_{t}\right)$ fully describes the optimization problem of timeslot $t$ of Model (7). To recognize this, let $\mathcal{R}_{D}$ be the set of $|\mathcal{E}|$ distinct dummy-rooms. I.e. for each event a dummy-room is created (and a corresponding edge is added to the graph) to ensure a matching of every event to a room will always exist. Hence the room-vertices of graph $G_{t}$ is given by $\overline{\mathcal{R}}=\mathcal{R} \cup \mathcal{R}_{D}$. The set of edges is given by (skipping edge definitions for the dummy-room vertices) $E_{t}=\left\{e \in \mathcal{E}, r \in \mathcal{R} \mid K_{e, r}=1 \wedge G_{r, t}=1\right\}$, and the weight on each edge is given by $\pi_{e, r}$. The goal of the matching problem is to select a maximum matching with minimum weight. A trivial maximum matching will assign every event to the dummy-room. Clearly this resembles component $t \in \mathcal{T}$ of Model (7).

Stating the Stage II model in terms of this graph allows us to exploit some well-known properties of matching problems in bipartite graphs. In the following, notation is simplified by dropping the $t$-index where applicable, i.e. we write $G$ instead of $G_{t}$ and $E$ instead of $E_{t}$. Denote by $\Gamma(S)$ the neighbors of event-nodes $S \subseteq \mathcal{E}$ in graph $G$, i.e. $\Gamma(S)=\{i \in \overline{\mathcal{R}} \mid j \in S,(i, j) \in E\}$. Hence $\Gamma(S) \subseteq \overline{\mathcal{R}}$. The well-known theorem of Hall states that a bipartite graph $G=(\mathcal{E} \cup \overline{\mathcal{R}}, E)$ has a matching of all vertices $\mathcal{E}$ into $\overline{\mathcal{R}}$ if and only if $|\Gamma(S)| \geq|S| \forall S \subseteq \mathcal{E}$. Observe that for timeslot $t \in \mathcal{T}$, the variable $y_{e, t}$ determines whether event $e \in \mathcal{E}$ is part of graph $G$. Lach and Lübbecke (2012) used this theorem to add constraints of the form

$$
\sum_{e \in S} y_{e, t} \leq|\Gamma(S)| \quad \forall S \subseteq \mathcal{E}, t \in \mathcal{T}
$$

to the Stage I model to guarantee that the Stage I model would yield a feasible matching problem for every component $t \in \mathcal{T}$ of the Stage II model. However, such constraints are redundant in our case, as we are guaranteeing that no matter how $y_{e, t}$ is selected, a feasible matching will always exist (due to the dummy-rooms). Instead we modify the expression (8) to provide a lower bound on the weighted matching problem. 
For the bipartite graph $G=(\mathcal{E} \cup \overline{\mathcal{R}}, E)$ (the edge-weights are set aside for now), let the deficiency of a vertex set $S \subseteq \mathcal{E}$ be defined as $\operatorname{def}(S)=|S|-|\Gamma(S)|$. Let the deficiency of $G$ be $\operatorname{defined}$ as $\operatorname{def}(G)=\max _{S \subseteq \mathcal{E}} \operatorname{def}(S)$. Theorem 1.3.1 of Lovász and Plummer (2009) states the following:

Theorem 4.1. The matching number of the bipartite graph $G=(\mathcal{E} \cup \overline{\mathcal{R}}, E)$, is $v(G)=|\mathcal{E}|-\operatorname{def}(G)$.

I.e. for the bipartite graph $G$, $\operatorname{def}(G)$ denotes the amount of vertices which are not matched in the maximum matching.

Let $\mathcal{W}$ denote the ordered set of different values found in $\pi_{e, r}$, i.e.

$$
\begin{aligned}
& \mathcal{W}=\left\{w \in \mathbb{R}^{+} \mid \exists e \in \mathcal{E}, \exists r \in \mathcal{R}: \pi_{e, r}=w\right\} \\
& w_{i}<w_{j} \Leftrightarrow \operatorname{ord}\left(w_{i}\right)<\operatorname{ord}\left(w_{j}\right) \quad \forall\left(w_{i}, w_{j}\right) \in \mathcal{W}
\end{aligned}
$$

Notice that no restrictions are posed on the amount of different values found, but it should be remarked that for our practical case, the cardinality of $\mathcal{W}$ is small (typically below 10 ).

The bipartite graph $G$ is split into subgraphs, one subgraph for each $w \in \mathcal{W}$. A subgraph is denoted as $G_{\leq w}=$ $\left(\mathcal{E} \cup \overline{\mathcal{R}}, E_{\leq w}\right)$, where the set of edges are those with at least weight $w$,

$$
E_{\leq w}=\left\{(e, r) \in E \mid \pi_{e, r} \leq w\right\}
$$

By these definitions, it is clear that

$$
\begin{aligned}
& \left|\Gamma\left(G_{\leq w_{1}}\right)\right| \leq\left|\Gamma\left(G_{\leq w_{2}}\right)\right| \leq \ldots \Rightarrow \\
& \operatorname{def}\left(G_{\leq w_{1}}\right) \geq \operatorname{def}\left(G_{\leq w_{2}}\right) \geq \ldots
\end{aligned}
$$

Using the deficiencies of these subgraphs, a lower bound on the minimum weight maximum matching can be stated. Let $a_{w} \in \mathbb{N}_{0}$ be defined as

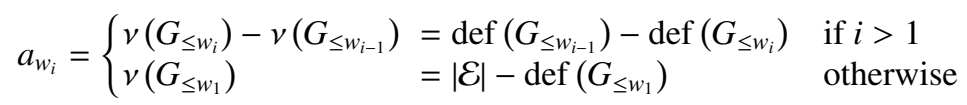

The intuition behind $a_{w}$ is to measure the change in the matching number when edges with weight $w$ are added to the subgraph $G_{\leq w_{i-1}}$. Note that $0 \leq a_{w} \leq|\mathcal{E}|$ for any $w \in \mathcal{W}$, as $0 \leq \operatorname{def}\left(G_{\leq w}\right) \leq|\mathcal{E}|$.

Theorem 4.2. The quantity

$$
\sum_{w \in \mathcal{W}} w \cdot a_{w}
$$

is a lower bound on a minimum weight maximum matching in the edge-weighted bipartite graph $G$.

Proof. Assume for contradiction there exists a maximum matching $M$ with lower weight, i.e.

$$
\sum_{e \in M} w_{e}<\sum_{w \in \mathcal{W}} w \cdot a_{w}
$$

Let $b_{w}$ denote the number of edges in $M$ of weight lesser or equal $w$, i.e.

$$
b_{w}=\left|\left\{e \in M: w_{e} \leq w\right\}\right|
$$

Let $k$ be the smallest number such that,

$$
b_{w_{k}}>\sum_{i=1}^{k} a_{w_{k}}
$$


This number must exist since $M$ is a cheaper matching. For the subgraph $G_{\leq w_{k}}, b_{w_{k}}$ can never exceed the matching number $v\left(G_{\leq w_{k}}\right)$ (by Theorem 4.1). We say 'exceed' as the matching might not include precisely $v\left(G_{\leq w_{k}}\right)$ edges of weight lesser or equal $w_{k}$. This gives:

$$
\begin{aligned}
b_{w_{k}} & \leq v\left(G_{\leq w_{k}}\right) \\
& =|\mathcal{E}|-\operatorname{def}\left(G_{\leq w_{k}}\right) \\
& =|\mathcal{E}| \underbrace{-\operatorname{def}\left(G_{\leq w_{1}}\right)+\operatorname{def}\left(G_{\leq w_{1}}\right)-\operatorname{def}\left(G_{\leq w_{2}}\right)+\operatorname{def}\left(G_{\leq w_{2}}\right) \ldots+\operatorname{def}\left(G_{\leq w_{k-1}}\right)}_{=0}-\operatorname{def}\left(G_{\leq w_{k}}\right) \\
& =\sum_{i=1}^{k} a_{w_{i}}
\end{aligned}
$$

which is a contradiction.

This lower bound is minimized in the objective of the Stage I model. Hence, any lower bound on the Stage I model is a lower bound on the overall problem. Additional notation is needed for stating the extended Stage I model.

The neighbors of event-nodes $S \subseteq \mathcal{E}$ in graph $G_{t, \leq w}$ is denoted $\Gamma_{t, \leq w}(S)$ for timeslot $t$ and weight $w$. Let the variable $\operatorname{def}_{t, \leq w} \in \mathbb{N}_{0}$ be the deficiency of subgraph $G_{t, \leq w}$. The deficiencies for each subgraph can be determined by adding the following constraint (follows directly from Theorem 4.1 and the definition of the deficiency for a bipartite graph),

$$
\sum_{e \in S} y_{e, t}-\operatorname{def}_{t, \leq w} \leq\left|\Gamma_{t, \leq w}(S)\right| \quad \forall S \subseteq \mathcal{E}, t \in \mathcal{T}, w \in \mathcal{W}
$$

Model (16) shows the extended model. Variables $\operatorname{def}_{t, \leq w}$ and $a_{t, w}$ are specified to be continuous as they will naturally take integer values. Obviously an exponential amount of constraints is added due to (16d), but it will be shown that for our practical purpose, the amount of required constraints is low.

Stage I extended with Hall's condition

$\min w^{I}=\sum_{e \in \mathcal{E}, t \in \mathcal{T}} \phi_{e, t} y_{e, t}+\sum_{t \in \mathcal{T}, w \in \mathcal{W}} w a_{t, w}$

s.t.

$$
\begin{array}{lll}
\text { (one timeslot) } \sum_{t \in \mathcal{T}} y_{e, t} & =1 & \forall e \in \mathcal{E} \\
\text { (resource conf.) } \sum_{e \in E_{a}^{\prime}} y_{e, t} & \leq 1 & \forall a \in \mathcal{A}, t \in \mathcal{T} \backslash t_{D} \\
& \sum_{e \in S} y_{e, t}-\operatorname{def}_{t, \leq w} \leq\left|\Gamma_{t, \leq w}(S)\right| \forall S \subseteq \mathcal{E}, t \in \mathcal{T}, w \in \mathcal{W} \\
\text { (Hall's) } & |\mathcal{E}|-\operatorname{def}_{t, \leq w_{1}}=a_{t, w_{1}} & \forall t \in \mathcal{T} \\
\text { (LB) } & \operatorname{def}_{t, \leq w_{-1}}-\operatorname{def}_{t, \leq w}=a_{t, w} & \forall t \in \mathcal{T}, w \in \mathcal{W}, \operatorname{ord}(w)>1 \\
\text { (LB) } & y_{e, t} \in\{0,1\} & \\
& \operatorname{def}_{t, \leq w}, a_{t, w} \in \mathbb{R}^{+} &
\end{array}
$$

Example 4.1. Below is shown an example of a bipartite graph and its subgraphs for some timeslot. Three different room-weights exists, $W=\{0,2,10\}$. Clearly, an optimal solution to the matching problem of this graph is $\left(e_{1}, r_{1}\right),\left(e_{2}, r_{3}\right),\left(e_{3}, r_{2}\right)$ with value 2 . The subgraphs for weights 0 and 2 are shown, and the lower bound derived. 


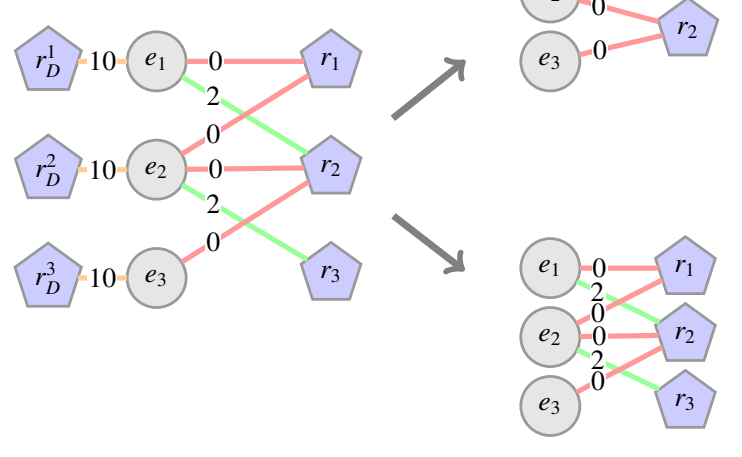

$$
\left.\begin{array}{l}
\begin{array}{l}
y_{e_{1}}-d e f_{\leq 0} \leq 1 \\
y_{e_{2}}-d e f_{\leq 0} \leq 2 \\
y_{e_{3}}-d e f_{\leq 0} \leq 1 \\
y_{e_{1}}+y_{e_{2}}-d e f_{\leq 0} \leq 2 \\
y_{e_{1}}+y_{e_{3}}-d e f_{\leq 0} \leq 2 \\
y_{e_{2}}+y_{e_{3}}-d e f_{\leq 0} \leq 2 \\
y_{e_{1}}+y_{e_{2}}+y_{e_{3}}-d e f_{\leq 0} \leq 2
\end{array} \\
y_{e_{1}}-d e f_{\leq 2} \leq 2 \\
y_{e_{2}}-d e f_{\leq 2} \leq 3 \\
y_{e_{3}}-d e f_{\leq 2} \leq 1 \\
y_{e_{1}}+y_{e_{2}}-d e f_{\leq 2} \leq 3 \\
y_{e_{1}}+y_{e_{3}}-d e f_{\leq 2} \leq 2 \\
y_{e_{2}}+y_{e_{3}}-d e f_{\leq 2} \leq 3 \\
y_{e_{1}}+y_{e_{2}}+y_{e_{3}}-d e f_{\leq 2} \leq 3
\end{array}\right\} \Rightarrow \begin{aligned}
& d e f_{\leq 0}=1 \\
& a_{0}=|\mathcal{E}|-d e f_{\leq 0}=3-1=2
\end{aligned}
$$

Hence the lower bound is derived as:

$$
L B=a_{0} 0+a_{2} 2=2
$$

Example 4.2. Naturally, the lower bound is not necessarily tight, as shown by the following small example.

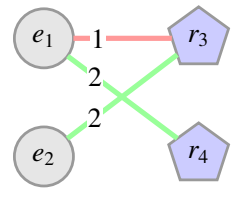

For weight $w_{1}=1$ the deficiency of $G_{1}$ is $\operatorname{def}\left(G_{1}\right)=1$ and therefore $a_{1}=1$. As the deficiency for $G_{2}$ is $\operatorname{def}\left(G_{2}\right)=0$ we also have $a_{2}=1$. The lower bound therefore reads $1 \cdot 1+2 \cdot 1=3$. But obviously the only (and therefore minimal weight) maximum matching has weight 4 . In fact, by increasing the weight on the weight 2 edges, it is seen that the gap between the lower bound and the actual minimal weight maximum matching could potentially be arbitrarily large. For the practical problem handled later, the weights can take values $\{1,2, \ldots, 10\}$, and therefore the gap between weights is low. The gap between the lower bound and the actual matchings obtained will be investigated experimentally.

\subsection{An exact approach using Egerváry's theorem}

An an alternative approach to the derived lower bound, the theorem of Egerváry (Egerváry (1931)) can be used to characterize the minimum weight of a matching in a bipartite graph, which deserves a mentioning in this context. The theorem states the following ((Schrijver, 2003, Theorem 17.1), here stated as a minimum weight problem):

Theorem 4.3. Let $G=(V, E)$ be a bipartite graph and let $w: E \rightarrow \mathbb{R}^{+}$be a weight function. Then the minimum weight of a matching in $G$ is equal to the maximum value of $y(V)$, where $y: V \rightarrow \mathbb{R}^{+}$is such that

$$
y_{u}+y_{v} \leq w_{e} \quad \forall u, v \in V,(u, v) \in E
$$

However, since we consider a bipartite graph for each timeslot, and each bipartite graph in worst case has $|\mathcal{E}||\mathcal{R}|$ vertices (which occurs often in practice), the amount of required constraints is of magnitude $|\mathcal{E}||\mathcal{R}||\mathcal{T}|$, so this is not a tractable approach. Furthermore, since the graph is not static (i.e. its structure depends on assignments of events to timeslots), a min - max formulation would be required. 


\subsection{Generating Hall's inequalities}

To generate the Hall's inequalities, it is necessary to exploit the structure of the underlying graph. I.e. we use problem specific knowledge to overcome the requirement of enumerating all subsets of events. In the Lectio case, two important features are known about the bipartite graphs:

- An event often has a special association with one specific room. This is either because the event is locked to that room, or because a penalty is imposed on not assigning an event to the room it was previously assigned to. In the later case, this means that one room has a lower weight than all other rooms for the particular event. Hence, in the subgraph $G_{\leq w}$ for this respective lower weight, only a single edge exists for the event. An event with only a single adjacent edge is denoted as a singleton event from now on.

- Furthermore, predefined feature-groups of rooms exist. A feature group of rooms is devoted to a certain type of lecture, for instance chemistry or physics, which require a room with specialized equipment. Hence many events are adjacent to the exact same set of rooms.

These graphs are hence exploited by separately considering the inequalities induced by singleton events and the inequalities induced by all other events, and finally those inequalities induced by combining these. The approach is formalized below. It should be remarked that applying this type of decomposition will require exploiting at least some properties of the underlying graph. We refer to Balas and Pulleyblank (1983), Edmonds (1965) and Lach and Lübbecke (2008) as helpful resources in this aspect.

For a subset of rooms $R \subseteq \mathcal{R}$, let $\Gamma^{-1}(R)$ be the set of events adjacent to only rooms in $R$, i.e. $\Gamma^{-1}(R)=$ $\{e \in \mathcal{E} \mid \Gamma(\{e\}) \subseteq R\}$.

Theorem 4.4. The Hall inequalities

$$
\sum_{e \in S} y_{e}-\operatorname{def} \leq|\Gamma(S)| \quad \forall S \subseteq \mathcal{E}
$$

are fully contained in

$$
\sum_{e \in \Gamma^{-1}(R)} y_{e}-\operatorname{def} \leq|R| \quad \forall R \subseteq \mathcal{R}
$$

Proof. Let $S \subseteq \mathcal{E}$ be any set of events. Now we let $R=\Gamma(S)$. Obviously we have $S \subseteq \Gamma^{-1}(R)=\Gamma^{-1}(\Gamma(S))$. If $\sum_{e \in \Gamma^{-1}(R)} y_{e}-\operatorname{def} \leq|R|$ holds we get

$$
\sum_{e \in S} y_{e}-\operatorname{def} \leq \sum_{e \in \Gamma^{-1}(R)} y_{e}-\operatorname{def} \leq|R|=|\Gamma(S)|
$$

This means that instead of having a constraint for every subset of events we can do with a constraint for every subset of rooms (which are considerably less).

Next we can further reduce the number of necessary constraints by exploiting symmetry between rooms. Rooms which are adjacent to exactly the same events can be grouped, and essentially treated as one room.

Theorem 4.5. Let $R_{1}, \ldots, R_{m} \subseteq \mathcal{R}$ be distinct $\left(i \neq j \Rightarrow R_{i} \cap R_{j}=\emptyset\right)$ subsets of rooms. Let $I \subseteq\{1, \ldots, m\}$ be an index set such that

$$
\bigcup_{i \in I} \Gamma^{-1}\left(R_{i}\right)=\Gamma^{-1}\left(\bigcup_{i \in I} R_{i}\right) \quad \text { (i.e. there is no event that only fits into a combination of the room sets in I) }
$$

Then the Hall constraint

$$
\sum_{e \in \Gamma^{-1}\left(\bigcup_{i \in I} R_{i}\right)} y_{e}-\operatorname{def} \leq\left|\bigcup_{i \in I} R_{i}\right|
$$




\section{is dominated by}

$$
\begin{aligned}
& \sum_{e \in R_{i}} y_{e}-\operatorname{def}_{i} \leq\left|R_{i}\right| \quad \forall i \in I \\
& \sum_{i \in I} \operatorname{def}_{i} \quad \leq \operatorname{def}
\end{aligned}
$$

where $\operatorname{def}_{i} \in \mathbb{N}_{0}$ is the deficiency of index $i \in I$, i.e. $\operatorname{def}_{i}=\operatorname{def}\left(\Gamma^{-1}\left(R_{i}\right)\right)$.

Proof. First note that $\sum_{i \in I} \operatorname{def}_{i} \leq$ def implies

$$
\sum_{e \in \Gamma^{-1}\left(\bigcup_{i \in I} R_{i}\right)} y_{e}-\operatorname{def} \leq \sum_{i \in I} \sum_{e \in \Gamma^{-1}\left(R_{i}\right)} y_{e}-\operatorname{def}_{i}
$$

Next by $\sum_{e \in \Gamma^{-1}\left(R_{i}\right)} y_{e}-\operatorname{def}_{i} \leq\left|R_{i}\right|$ we get

$$
\sum_{i \in I} \sum_{e \in \Gamma^{-1}\left(R_{i}\right)} y_{e}-\operatorname{def}_{i} \leq \sum_{i \in I}\left|R_{i}\right|=\left|\bigcup_{i \in I} R_{i}\right|
$$

where the later equality holds as the $R_{i}$ are distinct.

Since the amount of possible ways to select $I$ is exponential, this shows a potential way to limit the amount of necessary inequalities.

The graphs of the Lectio instances usually have the following structure, as previously discussed: Certain events are fixed to a specific room. These events are known as singleton events, and are denoted with the set $E^{1}$. If the singleton events are discarded, all other rooms can be grouped into groups $\mathcal{I}=\{1,2, \ldots, m\}$, i.e. $R_{i} \subseteq \mathcal{R} \forall i \in \mathcal{I}$, where every room is connected to the very same events as the other rooms of the same group. In particular this means

$$
\Gamma^{-1}(R) \backslash E^{1}=\emptyset \quad \forall R \subsetneq R_{i}, i \in \mathcal{I}
$$

I.e. no event is adjacent to only a subset of rooms of the room-groups, except for the singleton events. The key observation here is that the number of these groups of rooms is low, yielding a tractable way to generate the Hall inequalities. By Theorem 4.4 we know that a subset of rooms fully characterises one of the Hall constraints (and that it is sufficient to consider only those).

Corollary 4.1. Given the structure of the Lectio graphs, only the following subsets of rooms need to be considered wrt. eq. (15) (in the altered form defined by Theorem 4.4):

$$
\begin{array}{ll}
\text { (I) } \quad \Gamma(e) & \forall e \in E^{1} \\
\text { (II) } \bigcup_{i \in I} R_{i} & \forall I \subseteq \mathcal{I}
\end{array}
$$

Proof. For contradiction, let $\tilde{R} \subseteq \mathcal{R}$ be any other subset of rooms, i.e.

$$
\begin{array}{ll}
\tilde{R} \neq \Gamma(e) & \forall e \in E^{1} \\
\tilde{R} \neq \bigcup_{i \in I} R_{i} & \forall I \subseteq \mathcal{I}
\end{array}
$$

$\tilde{R}$ can be decomposed into subsets $\tilde{R}_{i}, i \in \mathcal{I}$, such that $\tilde{R}_{1} \subseteq R_{1}, \tilde{R}_{2} \subseteq R_{2}, \ldots, \tilde{R}_{m} \subseteq R_{m}$ and $\bigcup_{i \in \mathcal{I}} \tilde{R}_{i}=\tilde{R}$.

For each of the decomposed room sets $\tilde{R}_{i}$ we can now have one of the three following cases (by eq. (17), which disallows that $\tilde{R}_{i} \neq R_{i}$ and $\Gamma^{-1}\left(\tilde{R}_{i}\right) \backslash E^{1} \neq \emptyset$ ):

1. $\tilde{R}_{i}=R_{i}$

2. $\tilde{R}_{i} \neq R_{i}$ and $\Gamma^{-1}\left(\tilde{R}_{i}\right) \cap E^{1} \neq \emptyset$ 


$$
\text { 3. } \tilde{R}_{i} \neq R_{i} \text { and } \Gamma^{-1}\left(\tilde{R}_{i}\right)=\emptyset
$$

Let the respective indices be contained in the sets $I_{1}, I_{2}$ and $I_{3}$. The rooms from the third case $\left(\tilde{R}_{i}, i \in I_{3}\right)$ do not add events to the left hand side of a Hall constraint and can therefore be ignored.

If combining the rooms from the first case to $R^{\prime}=\bigcup_{i \in I_{1}} \tilde{R}_{i}$ we get one of the already considered combinations of room groups. Now note that there is no event fitting into the combination of $R^{\prime}$ with any of the rooms from the second case (their $\Gamma^{-1}$ only contains singleton events) and therefore the condition for Theorem 4.5 is met:

$$
\left(\bigcup_{i \in I_{2}} \Gamma^{-1}\left(\tilde{R}_{i}\right)\right) \cup \Gamma^{-1}\left(R^{\prime}\right)=\Gamma^{-1}\left(\left(\bigcup_{i \in I_{2}} \tilde{R}_{i}\right) \cup R^{\prime}\right)
$$

So we now know that the Hall constraint corresponding to $\tilde{R}$ is unnecessary.

Algorithm (4.1) shows the implemented algorithm for generation all necessary Hall constraints according to this construction.

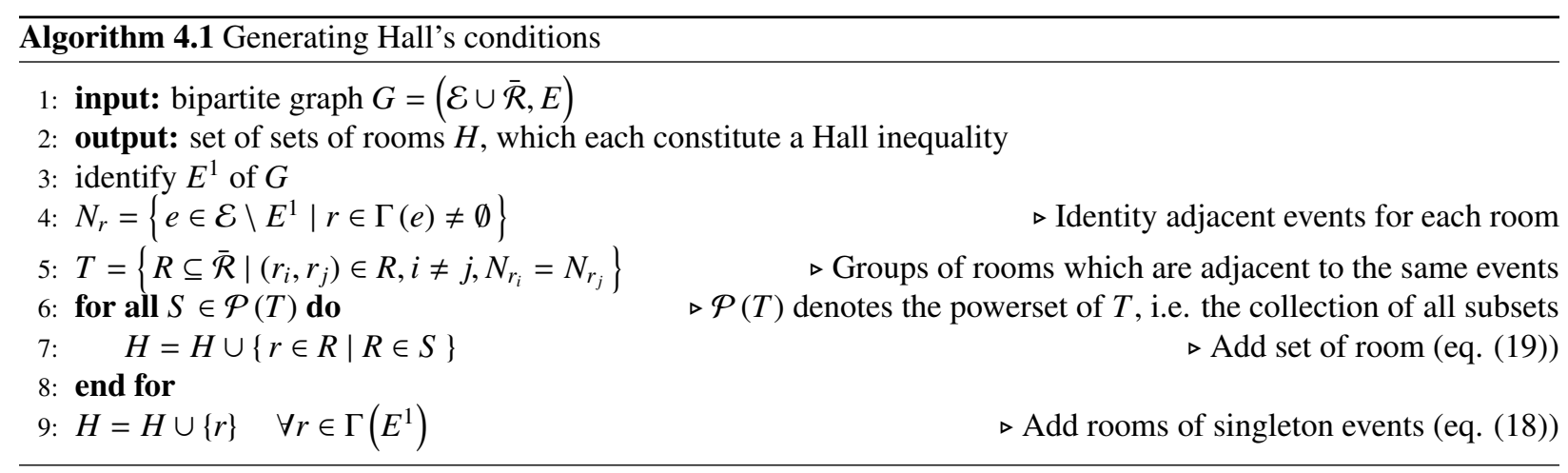

In Line 5 rooms are grouped. Here it should be remarked that this is done in a way that identifies the minimum number of groups of rooms. The amount of generated inequalities is hence exponential in the number of groups of rooms. For the Lectio high school timetabling problem, this number is in general low. However, an artificial limit of a maximum of 12 different groups of rooms is imposed, allowing in magnitude of $2^{12}$ inequalities to be generated for each timeslot. In practice, only two problem instances are restricted by this limit ("HasserG2012" and "SlagelG2012"). The room groups to generate inequalities are selected by ordering the room groups in terms of total number of adjacent events to all other room groups, and taking those room groups where this number is highest. Obviously, omitting some inequalities will not change the fact that the room allocation penalty added to Stage I is a lower bound on the objective of Stage II.

\section{Lectio High School Timetabling Problem}

To establish computational results, Stage I and Stage II are extended to the full version of the Danish case of high school timetabling described in Sørensen and Stidsen (2013). This variant of the problem is used in the timetabling component of the high school ERP-system Lectio, and hence reflects all aspects of a practical timetabling optimization problem. Lectio Timetabling is used by many high schools in Denmark, and this formulation of the problem has been used in production mode for over a year. In this paper a brief introduction to each of the added constraints and variables is given. More in-depth description and motivation can be found in Sørensen and Stidsen (2013).

This timetabling problem contains more types of constraints than what is usually found in the literature. This is mainly related to the big number of different high schools which use it, which inevitably gives a big variety of required features. However, a conversion scheme from this timetabling problem to the general XHSTT format is known, so the Lectio problem fits within the general concepts of high school timetabling problems.

Extensive computational experiments have shown that the usual formulation of this timetabling problem using a binary variable with three indices is very challenging for the commercial MIP solver Gurobi 5, which is among 
the very best general-purpose MIP solvers according to recent benchmarks of Mittelman (2013). Therefore this timetabling problem is a good candidate for testing the TSD approach.

\subsection{Stage I}

The set of timeslots $\mathcal{T}$ is defined by the combination of the set of days $\mathcal{D}$, and the set of daily-timeslots (known as modules) $\mathcal{M}$. The set of resources $\mathcal{A}$ consists of teachers and students, which is also known as the set of entities. Furthermore, the set of classes is denoted $C$. A class $c \in C$ is a non-physical resource treating a specific teachingsubject, and is associated with a certain set of events. Hence, an event can be viewed as a single lecture of a certain class. Parameter $J_{e, c} \in\{0,1\}$ takes value 1 if event $e \in \mathcal{E}$ is part of class $c \in C$, and 0 otherwise.

Variable $v_{a, t} \in\{0,1\}$ takes value 1 if entity $a \in \mathcal{A}$ is active in timeslot $t \in \mathcal{T}$, and 0 otherwise. Variable $f_{a, d} \in\{0,1\}$ takes value 1 if entity $a \in \mathcal{A}$ has no events scheduled on events on day $d \in \mathcal{D}$ (we say that the entity has a day off, even though he/she might be occupied by unscheduled activities, such as lecture preparation), and 0 otherwise. Variable $b_{c, t} \in\{0,1\}$ takes value 1 if class $c \in C$ has at least one lecture in timeslot $t \in \mathcal{T}$, and 0 otherwise. Variable $n_{c, d} \in\{0,1\}$ takes value if class $c \in C$ has a neighbor-day conflict on day $d \in \mathcal{D}$. A neighbor-day conflict occurs when the same class has scheduled events on two consecutive days. Variable $o_{a, d} \in\{0,1\}$ takes value 1 if entity $a \in \mathcal{A}$ has only one event on day $d \in \mathcal{D}$, and 0 otherwise. Days with only one lecture are undesirable and should be avoided. Variable $w_{c} \in \mathbb{N}_{0}$ is the amount which class $c \in C$ is 'out of week-balance'. I.e. if the set of timeslots is made up of times from more than one week, the amount of events of each class in each week must be equivalent (as far as possible). Variable $h_{a, d} \in \mathbb{N}_{0}$ is the amount of idle timeslots (a timeslot with no activity, but there exists both at least one earlier and one later timeslot with activity) for entity $a \in \mathcal{A}$ on day $d \in \mathcal{D}$. Variables $\underline{h}_{a, d}, \bar{h}_{a, d} \in \mathbb{N}_{0}$ denote the ordinal number of the first and last timeslot with activity on day $d \in \mathcal{D}$ for entity $a \in \mathcal{A}$, respectively.

The objective consists of 6 additional terms. These denote the weighted sum of entity idle slots (weight $\phi_{a} \in \mathbb{R}^{+}$), neighbor-day conflicts (weight $\zeta \in \mathbb{R}^{+}$), days with only one lecture (weight $\eta_{a} \in \mathbb{R}^{+}$), days-off for teachers (weight $\gamma_{a} \in \mathbb{R}^{+}$), days-off for students (weight $\delta_{a} \in \mathbb{R}^{+}$), and class week stability (weight $\iota \in \mathbb{R}^{+}$), respectively.

Let parameters $S_{e}$ and $C_{e}$ be the set of events which should be scheduled in the same timeslot as event $e \in \mathcal{E}$, and in the timeslot immediately following event $e \in \mathcal{E}$, respectively. Parameter $P_{d, d^{\prime}} \in\{0,1\}$ takes value 1 if day $d \in \mathcal{D}$ and day $d^{\prime} \in \mathcal{D}$ are neighbor-days, and 0 otherwise. Parameter $R_{c, d} \in\{0,1\}$ takes value 1 if class $c \in C$ is part of some event which is locked to some timeslot on day $d \in \mathcal{D}$, and let $N_{c} \in \mathbb{N}_{0}$ be the number of allowed neighbor-day conflicts for class $c \in C$. $\mathcal{T}_{d}$ denotes the set of timeslots on day $d \in \mathcal{D}$. Parameter $D_{e, t} \in\{0,1\}$ takes value 1 if event $e \in \mathcal{E}$ can be scheduled in timeslot $t \in \mathcal{T}$, and 0 otherwise. Parameter $F_{a} \in \mathbb{N}_{0}$ denotes the amount of required days off for entity $a \in \mathcal{A}$. Parameter $W_{a} \in \mathbb{N}_{0}$ denotes the maximum amount of events which can be scheduled to entity $a \in \mathcal{A}$ on any given day.

A class can only have one event assigned to each day, unless it is specified that multiple events should be placed in contiguous positions. We say that such day-conflicts are infeasible. The set $E^{\prime \prime \prime} \subseteq \mathcal{E}$ denotes the set of events for which day-conflicts are checked.

The most common case is that a school creates a timetable for a single week. However, some schools desire to create a two-week timetable instead. This allows more flexibility in the planning; take for instance a class with a nominated teaching load of three events per week. In case the school uses a two-week timetable, this class can for instance have one double lecture in the first week, and two double lectures in the second week. $d(\underline{\mathcal{T}})$ and $d(\overline{\mathcal{T}})$ denotes the set of days in the first and in the second week, respectively. $\underline{\mathcal{T}}$ and $\overline{\mathcal{T}}$ denotes the timeslots in the first and second week, respectively.

The complete Stage I model is shown in (20). 


$$
\begin{aligned}
& \min w^{I}=\sum_{e \in \mathcal{E}, t \in \mathcal{T}} \phi_{e, t} y_{e, t}+\sum_{t \in \mathcal{T}, w \in \mathcal{W}} w a_{t, w}+\sum_{a \in \mathcal{A}, d \in \mathcal{D}} \beta_{a} h_{a, d}+\zeta \sum_{c \in \mathcal{C}, d \in \mathcal{D}} n_{c, d}+\sum_{a \in \mathcal{A}, d \in \mathcal{D}} \eta_{a} o_{a, d} \\
& \text { s.t. } \left.\quad+\sum_{a \in \mathcal{A}} \gamma_{a}|| \mathcal{D} \mid-\sum_{d \in \mathcal{D}} f_{a, d}\right]+\sum_{a \in \mathcal{A}, d \in \mathcal{D}} \delta_{a} f_{a, d}+\iota \sum_{c \in \mathcal{C}} w_{c} \\
& \text { (one timeslot) } \quad \sum_{t \in \mathcal{T}} y_{e, t} \\
& \text { (entity time aux.) } \sum_{e \in E_{a}^{\prime}} y_{e, t} \\
& \begin{array}{ll}
\text { (entity conf.) } & \sum_{t \in \mathcal{T}_{d}} v_{a, t}+f_{a, d} \\
\text { (Hall's) } & \sum_{e \in S} y_{e, t}-\operatorname{def}_{t, \leq w}
\end{array} \\
& =1 \quad \forall e \in \mathcal{E} \\
& =v_{a, t} \quad \forall a \in \mathcal{A}, t \in \mathcal{T} \\
& \leq 1 \quad \forall a \in \mathcal{A}, d \in \mathcal{D} \\
& \leq\left|\Gamma_{t, \leq w}(S)\right| \forall S \subseteq \mathcal{E}, t \in \mathcal{T}, w \in \mathcal{W} \\
& \text { (room alloc. lb) }|\mathcal{E}|-\operatorname{def}_{t, \leq w_{1}} \\
& \text { (room alloc. lb) } \operatorname{def}_{t, \leq w_{-1}}-\operatorname{def}_{t, \leq w} \\
& \text { (locked time) } \quad y_{e, t} \\
& \text { (same time) } \quad y_{e, t}-y_{e^{\prime}, t} \\
& \text { (cont. times) } \quad y_{e, t}-y_{e^{\prime}, t^{\prime}} \\
& \text { (n.d. conf.) } \quad \sum_{t \in \mathcal{T}_{d}} b_{c, t}+\sum_{t \in \mathcal{T}_{d^{\prime}}} b_{c, t}-n_{c, d} \\
& \text { (n.d. conf.) } \quad \sum_{d \in \mathcal{D}} n_{c, d} \\
& \text { (forbid. times) } \sum_{t \in \mathcal{T}, D_{e, t}=0} y_{e, t} \\
& =a_{t, w_{1}} \quad \forall t \in \mathcal{T} \\
& =a_{t, w} \quad \forall t \in \mathcal{T}, w \in \mathcal{W}, \operatorname{ord}(w)>1 \\
& =1 \quad \forall e \in \mathcal{E}, t \in \mathcal{T}, L T_{e, t}=1 \\
& =0 \quad \forall e \in \mathcal{E}, e^{\prime} \in S_{e}, t \in \mathcal{T} \\
& =0 \quad \forall e \in \mathcal{E}, e^{\prime} \in C_{e},\left(t, t^{\prime}\right) \in \mathcal{T}, d_{t}=d_{t^{\prime}}, \\
& \operatorname{ord}(t)+1=\operatorname{ord}\left(t^{\prime}\right) \\
& \leq 1 \quad \forall c \in \mathcal{C},\left(d, d^{\prime}\right) \in \mathcal{D}, P_{d, d^{\prime}}=1, \\
& R_{c, d}+R_{c, d^{\prime}} \leq 1 \\
& \leq N_{c} \quad \forall c \in C \\
& =0 \quad \forall e \in \mathcal{E} \\
& =h_{a, d} \quad \forall a \in \mathcal{A}, d \in \mathcal{D} \\
& \begin{array}{llll}
\text { (idle slots) } & |\mathcal{M}|-(|\mathcal{M}|-\operatorname{ord}(t)) v_{a, t} & \geq \underline{h}_{a, d} & \forall a \in \mathcal{A}, d \in \mathcal{D}, t \in \mathcal{T}_{d} \\
\text { (idle slots) } & \operatorname{ord}(t) v_{a, t} & \leq \bar{h}_{a, d} & \forall a \in \mathcal{A}, d \in \mathcal{D}, t \in \mathcal{T}_{d}
\end{array} \\
& \text { (days off) } \quad \sum_{d \in \mathcal{D}} f_{a, d} \\
& \geq F_{a} \quad \forall a \in \mathcal{A} \\
& \geq 1 \quad \forall a \in \mathcal{A}, d \in \mathcal{D} \\
& \text { (days off) } \quad \sum_{t \in \mathcal{T}_{d}} v_{a, t}+f_{a, d} \\
& \text { (day conf.) } \quad \sum_{e \in E^{\prime \prime \prime}} J_{e, c} y_{e, t} \\
& \text { (day conf.) } \quad \sum_{t \in \mathcal{T}_{d}}^{e \in E^{\prime \prime}} b_{c, t} \\
& \text { (work limit) } \sum_{e \in \mathcal{E}, t \in \mathcal{T}_{d}} y_{e, t} \\
& \text { (one lecture) } \quad 2-\sum_{t \in \mathcal{T}_{d}} v_{a, t}-2 f_{a, d} \quad \leq o_{a, d} \quad \forall a \in \mathcal{A}, d \in \mathcal{D} \\
& \text { (class stabl.) }\left|\sum_{e \in \mathcal{E}, t \in \mathcal{T}} J_{e, c} y_{e, t}-\sum_{e \in \mathcal{E}, t \in \overline{\mathcal{T}}} J_{e, c} y_{e, t}\right|-1=w_{c} \quad \forall c \in C \\
& \text { (d.o. stabl.) }\left|\sum_{d \in d(\mathcal{T})} f_{a, d}-\sum_{d \in d(\overline{\mathcal{T}})} f_{a, d}\right| \quad \leq 1 \quad \forall a \in \mathcal{A} \\
& \leq b_{c, t} \quad \forall c \in C, t \in \mathcal{T} \\
& \leq 1 \quad \forall c \in C, d \in \mathcal{D} \\
& \leq W_{a} \quad \forall a \in \mathcal{A}, d \in \mathcal{D} \\
& y_{e, t} \quad \in\{0,1\} \\
& v_{a, t}, f_{a, d}, b_{c, t}, n_{c, d}, o_{a, d} \quad \in[0,1] \\
& h_{a, d}, \underline{h}_{a, d}, \bar{h}_{a, d}, w_{c}, \operatorname{def}_{t, \leq w}, a_{t, w} \quad \in \mathbb{R}^{+}
\end{aligned}
$$


Constraint (20c) constraints the auxiliary variable $v_{a, t}$ properly. Constraint (20d) treats entity conflicts in a slightly changed formulation, to also constrain variable $f_{a, d}$ properly. Constraints (20e)-(20g) define the lower bound on room allocation, and are similar to those previously described. Constraint (20h) ensures the assigning of events are locked to a certain timeslot. Constraints $(20 \mathrm{i})$ and $(20 \mathrm{j})$ ensure the placement of events which must be placed in the same/contiguous timeslots. Constraints $(20 \mathrm{k})$ and $(201)$ ensure that variable $n_{c, d}$ is constrained properly, and that no more than $N_{c}$ neighbor-day conflicts are scheduled for class $c \in C$. Constraint (20m) poses restrictions on timeslots for which an entity is unavailable. Constraints (20n)-(20p) ensures that idle slots for entities are penalized accordingly. Constraint (20q) ensures that sufficient days off is assigned to each entity. Constraint (20r) makes sure that if an entity $a \in \mathcal{A}$ has no event on some day $d \in \mathcal{D}$, then variable $f_{a, d}$ is forced to take value 1 . This is necessary as this variable is minimized in the objective. Constraints (20s) and (20t) ensure that day-conflicts for classes does not occur, and constraints the variable $b_{c, t}$ properly. Constraint $(20 \mathrm{u})$ ensures that the limit on the amount of events assigned to a day for entity $a$ is respected. For an entity, days with only one event scheduled are undesirable. Constraint (20v) penalizes days with only one events scheduled for entity $a$. Constraint $(20 \mathrm{w})$ forces week-stability for events of classes, i.e. in case two-weeks are being planned, events for courses must be spread evenly throughout the weeks. Constraint (20x) ensures that in case several weeks are being planned, the days off for an entity are spread evenly throughout the weeks.

\subsection{Stage II}

Let variable $v_{c, r} \in\{0,1\}$ take value 1 if there is at least one event of which class $c \in C$ participates assigned to room $r \in \mathcal{R}$, and 0 otherwise. Variable $s_{c} \in \mathbb{N}_{0}$ is the amount of 'excess' rooms assigned to events of class $c \in C$, i.e. the total amount of rooms assigned minus one. This is used to enforce room stability for classes, since it is undesirable for a class to be assigned too many different rooms. Parameter $L R_{e, r} \in\{0,1\}$ takes value 1 if event $e \in \mathcal{E}$ is locked room $r \in \mathcal{R}$.

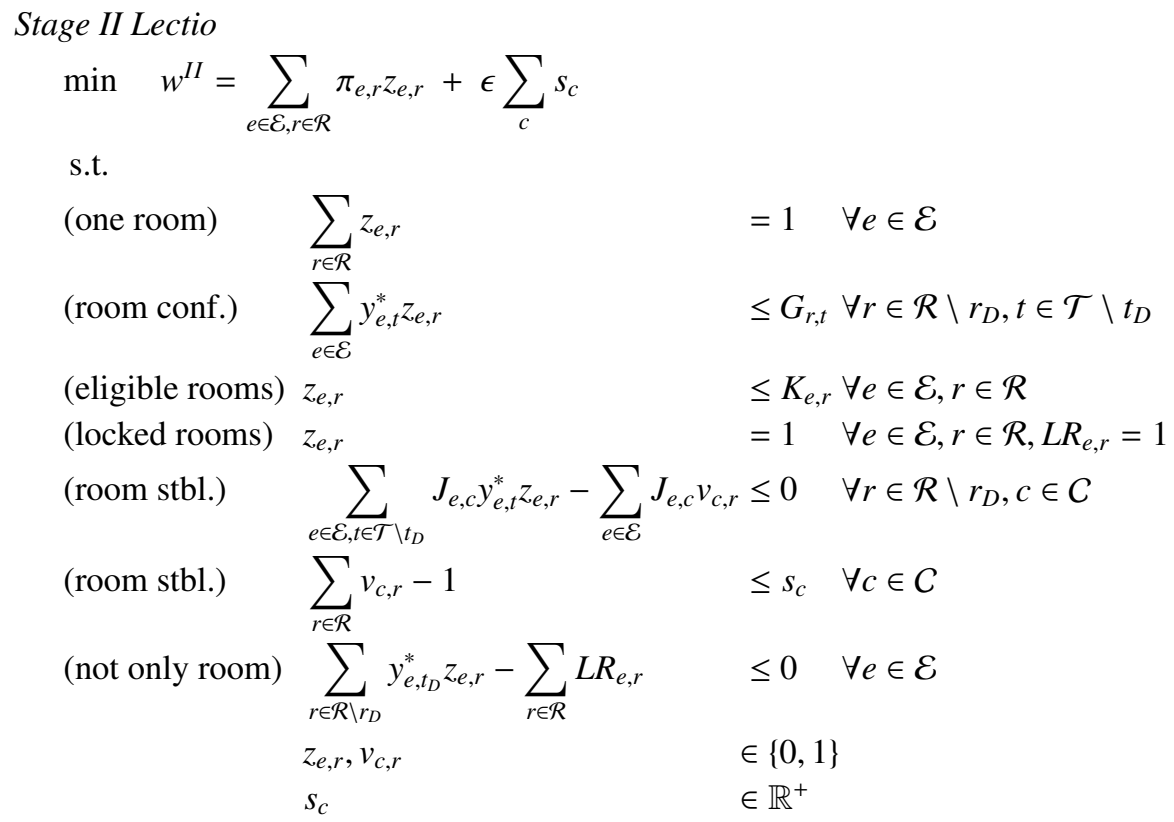

Constraint (21e) ensures that events with locked rooms are assigned accordingly. Constraints (21f) and (21g) constraints variables $v_{c, r}$ and $s_{c}$ properly, and thereby penalizes room stability. Constraint (21h) enforces that an event cannot be assigned a room if it not assigned to a timeslot, unless the event is locked to a specific room.

Notice that the room stability constraints (21f) and (21g) of Model (21) are not handled in any way in the Stage I model. Additional constraints which handle these constraints would theoretically improve the decomposition. It is however not trivial to model these constraints as a matching problem in a bipartite graph, so another approach may be required. This is a subject for future research. Apart from the room stability constraints, all other constraints are 
optimally handled by the decomposition, with the exception of the room allocation penalties, which are only partially integrated in the Stage I model.

\section{Computational Results}

For implementation purposes, Gurobi 5.0.1 was used as MIP solver on a machine with an Intel Core i7 930@2.80GHz CPU and 12GB of RAM, running Windows 8 64bit. Default parameter settings were used, and the interface was C\# 4.5. The problem instances have been taken directly from the Lectio database, and are the same ones used in Sørensen and Stidsen (2013). These 100 real-world datasets provide a substantial ground for concluding on the numerical experiments. Note that 3 of these instances are available in the XHSTT format (Post et al. (2012a)) at http://www.utwente.nl/ctit/hstt/. We plan to make additional datasets available in this format in the future.

A time limit of 7200 seconds was imposed (6480 seconds for Stage I, and 720 seconds for Stage II), and Gurobi was allowed to use 8 threads. For the Stage I model, the initial solution given to Gurobi consists of assigning all events to the dummy-timeslot, except for those events locked to a specific timeslot. The initial solution for the Stage II model is analogous; Events are assigned to the dummy-room or the room which the event is locked too. A single run was used to establish results, as Gurobi has deterministic behavior.

Two other solution approaches are described for the same timetabling problem in Sørensen and Stidsen (2013). These are used in comparison with the algorithm of this paper, and are briefly described below:

- The 'usual' formulation using a three-index binary variable, denoted 3-index model in the following. This is solved using Gurobi with standard settings, with a time limit of 7200 seconds. The objectives listed are the result of a single run.

- An Adaptive Large Neighborhood Search heuristic, denoted ALNS in the following. The reported objectives for this method is the average obtained over 10 runs, each run with a timelimit of 240 seconds. Hence, the comparison of objectives wrt. this heuristic is not 'fair', but it will be seen that even with this shorter timelimit, the ALNS in general performs best. This method is the one currently used by the customers of Lectio.

Furthermore, we test the described decomposition both with and without the room penalties added to the Stage 1 model (i.e. Model (20) with and without equations (20e), (20f) and (20g)). Thereby an empirical test of the effect of extending Stage I is performed. In the following, these two methods are denoted TSD and TSD ${ }^{\text {RoomLB }}$, respectively. Notice that the results for TSD can also be found in the technical report Sørensen and Stidsen (2013).

Table 1 shows the obtained results. Table 2 summarizes some key numbers. Table 3 gives a summary for the three exact methods. A gap between an IP objective $z$ and a lower bound LB is calculated by $100 \frac{z-L B}{z}$.

Table 1: Computational results. For each dataset is shown the objective 'Obj' obtained by each method. For the IP-based approaches, also the best found lower bounds 'LB' are shown (i.e. for the 3-index model is shown the final value of the LP-relaxation used internally by Gurobi, and for TSD is shown the final value of the LP-relaxation of the Stage I model, as described in Section 4). If a solution is best overall, it is marked in bold. If a bound is best overall, it is marked with a '*'. For the two-stage approach of this paper is shown the runtime 'Time' and final gap 'Gap' found by Gurobi for both Stage I and Stage II. For Stage II, column 'Diff.' denotes the difference between the lower bound for room allocation of Stage I, and the actual matching obtained by Stage II (excluding room stability). Column ' $\overline{\text { Gap }}$, denotes the best overall gap, i.e. the gap between the best available solution and the best available bound.

\begin{tabular}{|c|c|c|c|c|c|c|c|c|c|c|c|c|c|}
\hline \multirow[b]{3}{*}{ Dataset } & \multicolumn{5}{|c|}{ Previous methods } & \multicolumn{8}{|c|}{$\mathrm{TSD}^{\text {RoomLB }}$} \\
\hline & \multirow{2}{*}{$\frac{\text { ALNS }}{\text { Obj }}$} & \multicolumn{2}{|c|}{ 3-index model } & \multicolumn{2}{|c|}{ TSD } & \multicolumn{2}{|c|}{ Stage I } & \multicolumn{3}{|c|}{ Stage II } & \multirow[b]{2}{*}{ Obj } & \multirow[b]{2}{*}{ LB } & \multirow[b]{2}{*}{$\overline{\text { Gap }}$} \\
\hline & & Obj & LB & Obj & LB & Time & Gap & Time & Gap & Diff. & & & \\
\hline AalborTG2012 & 6317 & 6118 & *5946 & 6018 & 5934 & $>6480$ & 0.7 & 4 & 0.0 & 0 & 6005 & 5941 & 1.0 \\
\hline AarhusA2011 & 10037 & 58015 & - & 15872 & $* 5986$ & $>6480$ & 66.6 & 154 & 0.0 & 160 & 18122 & 5985 & 40.4 \\
\hline AarhusA2012 & 7971 & 17096 & 5722 & 8947 & $* 6005$ & $>6480$ & 49.4 & 108 & 0.0 & 48 & 11936 & 5962 & 24.7 \\
\hline Aars2009 & 14900 & 49504 & - & 20780 & 11874 & $>6480$ & 47.9 & 14 & 0.0 & 0 & 24240 & $* 12641$ & 15.2 \\
\hline Aars2010 & 16268 & 81970 & - & 25057 & 13134 & $>6480$ & 42.7 & 22 & 0.0 & 1 & 24692 & $* 14151$ & 13.0 \\
\hline Aars2011 & 14256 & 77967 & - & 30623 & 9709 & $>6480$ & 68.9 & 10 & 0.0 & 3 & 33790 & $* 10501$ & 26.3 \\
\hline
\end{tabular}


Table 1 - continued from previous page

Previous methods

$\mathrm{TSD}^{\text {RoomLB }}$

\begin{tabular}{|c|c|c|c|c|c|c|c|c|c|c|c|c|c|}
\hline \multirow[b]{3}{*}{ Dataset } & \multirow{3}{*}{$\frac{\text { ALNS }}{\text { Obj }}$} & \multirow{2}{*}{\multicolumn{2}{|c|}{ 3-index model }} & \multirow{2}{*}{\multicolumn{2}{|c|}{ TSD }} & \multirow{2}{*}{\multicolumn{2}{|c|}{ Stage I }} & \multirow{2}{*}{\multicolumn{3}{|c|}{ Stage II }} & \multirow[b]{3}{*}{ Obj } & \multirow[b]{3}{*}{ LB } & \multirow[b]{3}{*}{$\overline{\text { Gap }}$} \\
\hline & & & & & & & & & & & & & \\
\hline & & Obj & LB & Obj & LB & Time & Gap & Time & Gap & Diff. & & & \\
\hline Aars2012 & 10701 & 55049 & - & 21206 & 7456 & $>6480$ & 60.3 & 21 & 0.0 & 1 & 20274 & $* 8044$ & 24.8 \\
\hline Alssund 2010 & 9967 & 52717 & - & 23173 & 6811 & $>6480$ & 67.9 & 324 & 0.0 & 8 & 21455 & $* 6876$ & 31.0 \\
\hline Alssund 2012 & 29803 & 108810 & - & 108810 & - & $>6480$ & - & 6 & 0.0 & 0 & 108810 & - & - \\
\hline BagsvaG2010 & 3960 & 6777 & 3171 & 3916 & 3063 & $>6480$ & 19.4 & 14 & 0.0 & 9 & 4051 & $* 3227$ & 17.6 \\
\hline BirkerG2011 & 42063 & 119600 & - & 119600 & - & $>6480$ & - & 7 & 0.0 & 0 & 119600 & - & - \\
\hline BirkerG2012 & 19552 & 110180 & - & 19322 & 15662 & $>6480$ & 0.9 & $>720$ & 1.0 & 16 & 18182 & $* 17709$ & 2.6 \\
\hline BjerrG2009 & 16877 & 52639 & - & 35514 & 11094 & $>6480$ & 55.2 & 11 & 0.0 & 0 & 27396 & *12288 & 27.2 \\
\hline BjerrG2010 & 4983 & 12868 & *3928 & 5788 & 3868 & $>6480$ & 33.1 & 13 & 0.0 & 37 & 5977 & 3925 & 21.2 \\
\hline BjerrG2011 & 6334 & 13009 & $* 4142$ & 9302 & 4060 & $>6480$ & 64.8 & 97 & 0.0 & 20 & 11676 & 4079 & 34.6 \\
\hline BjerrG2012 & 8023 & 17200 & $* 5055$ & 15265 & 5007 & $>6480$ & 71.2 & 160 & 0.0 & 16 & 17404 & 4991 & 37.0 \\
\hline BroendG2012 & 2040 & 2005 & $* 1881$ & 1929 & 1859 & 1028 & 0.0 & 17 & 0.0 & 5 & 1928 & 1877 & 2.4 \\
\hline CPHWGym2010 & 6775 & 34415 & - & 19363 & $* 3759$ & $>6480$ & 77.4 & 11 & 0.0 & 0 & 16589 & 3752 & 44.5 \\
\hline CPHWGym2011 & 5679 & 38232 & - & 16212 & 4095 & $>6480$ & 72.7 & 10 & 0.0 & 0 & 15046 & $* 4103$ & 27.8 \\
\hline CPHWGym2012 & 6762 & 40945 & - & 15543 & 4205 & $>6480$ & 75.5 & 19 & 0.0 & 1 & 17194 & $* 4215$ & 37.7 \\
\hline CPHWHG2012 & 11077 & 46625 & 8157 & 23088 & $* 8338$ & $>6480$ & 64.1 & 16 & 0.0 & 0 & 23219 & 8326 & 24.7 \\
\hline СРHWHTX2010 & 11342 & 27174 & 9179 & 15943 & 8828 & $>6480$ & 52.1 & 7 & 0.0 & 0 & 19314 & $* 9259$ & 18.4 \\
\hline СРНWHTX2011 & 20734 & 22466 & 20460 & 20708 & 18490 & $>6480$ & 0.6 & 6 & 0.0 & 11 & 20632 & $* 20470$ & 0.8 \\
\hline СРHWHTX2012 & 16256 & 25998 & 14481 & 21392 & 13115 & $>6480$ & 35.4 & 4 & 0.0 & 0 & 22481 & $* 14531$ & 10.6 \\
\hline DetFG2012 & 7560 & 8017 & *7168 & 7265 & 7018 & $>6480$ & 0.7 & 8 & 0.0 & 68 & 7258 & 7116 & 1.2 \\
\hline DetKG2010 & 2947 & 6058 & 1732 & 4006 & $* 1821$ & $>6480$ & 55.6 & 3 & 0.0 & 4 & 4102 & 1814 & 38.2 \\
\hline DetKG2011 & 2820 & 5594 & 1732 & 4366 & 1780 & $>6480$ & 60.9 & 2 & 0.0 & 2 & 4577 & $* 1781$ & 36.8 \\
\hline EUCN2009 & 3737 & 7557 & 2911 & 4298 & 2856 & $>6480$ & 40.3 & 4 & 0.0 & 0 & 5001 & *2982 & 20.2 \\
\hline EUCN2010 & 3882 & 4231 & 3329 & 3463 & 3246 & $>6480$ & 1.4 & 6 & 0.0 & 1 & 3430 & $* 3375$ & 1.6 \\
\hline EUCN2011 & 1468 & 1435 & *1395 & 1430 & 1384 & $>6480$ & 2.2 & 1 & 0.0 & 2 & 1426 & 1384 & 2.2 \\
\hline EUCN2012 & 3289 & 9430 & 2327 & 5059 & $* 2363$ & $>6480$ & 60.2 & 4 & 0.0 & 0 & 5913 & 2359 & 28.2 \\
\hline EUCNHG2010 & 1505 & 1476 & 1371 & 1421 & 1368 & $>6480$ & 2.1 & 2 & 0.0 & 0 & 1408 & $* 1378$ & 2.1 \\
\hline EUCS2012 & 3714 & 4689 & 3576 & 3783 & 3347 & $>6480$ & 3.0 & 3 & 0.0 & 0 & 3695 & $* 3584$ & 3.0 \\
\hline FaaborgG2008 & 68124 & 125330 & - & 125330 & - & $>6480$ & - & 14 & 0.0 & 0 & 125330 & - & - \\
\hline FalkonG2009 & 10449 & 88890 & - & 88890 & - & $>6480$ & - & 5 & 0.0 & 0 & 88890 & - & - \\
\hline FalkonG2011 & 8584 & 76170 & - & 16543 & $* 5183$ & $>6480$ & 75.9 & $>720$ & 0.0 & 48 & 20758 & 4953 & 39.6 \\
\hline FalkonG2012 & 10143 & 100190 & - & 16666 & $* 6105$ & $>6480$ & 58.6 & $>720$ & 0.1 & 121 & 14908 & 6050 & 39.8 \\
\hline GUAasia2010 & 6527 & 6579 & 6354 & 6461 & 6035 & 26 & 0.0 & $>720$ & 0.1 & 5 & 6422 & $* 6374$ & 0.7 \\
\hline GUQaqor2011 & 6674 & 19623 & 4537 & 10005 & $* 4554$ & $>6480$ & 59.9 & 3 & 0.0 & 18 & 11396 & 4542 & 31.8 \\
\hline GUQaqor2012 & 5733 & 11488 & 4314 & 7619 & 4294 & $>6480$ & 55.1 & 10 & 0.0 & 0 & 9650 & $* 4324$ & 24.6 \\
\hline HadersK2011 & 7128 & 51190 & - & 14229 & *3909 & $>6480$ & 76.2 & $>720$ & 0.0 & 43 & 16494 & 3888 & 45.2 \\
\hline HasserG2010 & 11963 & 96790 & - & 96790 & - & $>6480$ & - & 6 & 0.0 & 0 & 96790 & - & - \\
\hline HasserG2011 & 16061 & 99840 & - & 99840 & - & $>6480$ & - & 6 & 0.0 & 0 & 99840 & - & - \\
\hline HasserG2012 ${ }^{\dagger}$ & 18338 & 112160 & - & 112034 & - & $>6480$ & - & 7 & 0.0 & 0 & 112160 & - & - \\
\hline HerningG2010 & 37 & 37 & $* 37$ & 37 & 35 & 0 & 0.0 & 1 & 0.0 & 0 & 37 & 35 & 0.0 \\
\hline HerningG2011 & 15091 & 163785 & - & 23117 & $* 9829$ & $>6480$ & 61.8 & 108 & 0.0 & 169 & 26410 & 9746 & 34.9 \\
\hline HerningG2012 & 13147 & 185433 & - & 14952 & 9763 & $>6480$ & 48.4 & $>720$ & 0.1 & 262 & 19834 & $* 9817$ & 25.3 \\
\hline HoejeTaG2008 & 2958 & 6292 & 2253 & 2707 & 2563 & $>6480$ & 6.4 & 3 & 0.0 & 0 & 2775 & $* 2587$ & 4.4 \\
\hline HoejeTaG2009 & 9157 & 45260 & - & 26066 & $* 5773$ & $>6480$ & 79.7 & 105 & 0.0 & 3 & 27779 & 5628 & 37.0 \\
\hline HoejeTaG2010 & 9862 & 45095 & - & 25678 & $* 6188$ & $>6480$ & 78.1 & 106 & 0.0 & 5 & 27886 & 6116 & 37.3 \\
\hline HoejeTaG2011 & 10158 & 51050 & - & 32630 & $* 6726$ & $>6480$ & 78.2 & 66 & 0.0 & 3 & 30327 & 6601 & 33.8 \\
\hline HoejeTaG2012 & 12502 & 72455 & 7592 & 18627 & 7845 & $>6480$ & 79.8 & 9 & 0.0 & 3 & 39326 & $* 7952$ & 36.4 \\
\hline HorsenS2009 & 3111 & 3100 & $* 3100$ & 3100 & 2865 & 1 & 0.0 & 4 & 0.0 & 13 & 3100 & 3059 & 0.0 \\
\hline HorsenS2012 & 10056 & 86090 & - & 86090 & - & $>6480$ & - & 3 & 0.0 & 0 & 86090 & - & - \\
\hline Johann2012 & 23001 & 92575 & - & 27781 & 18456 & $>6480$ & 33.5 & 233 & 0.0 & 6 & 29491 & *19590 & 14.8 \\
\hline KalundG2011 & 38479 & 126150 & - & 126150 & - & $>6480$ & - & 9 & 0.0 & 0 & 126150 & - & - \\
\hline KalundG2012 & 26768 & 123010 & - & 123010 & - & $>6480$ & - & 11 & 0.0 & 0 & 123010 & - & - \\
\hline KalundHG2010 & 5631 & 12103 & 4540 & 6351 & 4551 & $>6480$ & 29.7 & 6 & 0.0 & 0 & 6605 & $* 4642$ & 17.6 \\
\hline KoebenPG2012 & 888 & 1872 & 637 & 874 & 642 & $>6480$ & 37.9 & 1 & 0.0 & 2 & 1052 & *645 & 26.2 \\
\hline KoegeH2012 & 11418 & 108347 & - & 20150 & 9096 & $>6480$ & 53.7 & 12 & 0.0 & 0 & 20390 & $* 9440$ & 17.3 \\
\hline KongshoG2010 & 4296 & 8889 & 2411 & 7954 & $* 2488$ & $>6480$ & 65.9 & 30 & 0.0 & 0 & 7208 & 2451 & 42.1 \\
\hline MariageG2009 & 8013 & 54030 & - & 20138 & 5118 & $>6480$ & 69.7 & $>720$ & 0.0 & 18 & 17506 & $* 5286$ & 34.0 \\
\hline MorsoeG2012 & 5651 & 42762 & - & 10241 & 3854 & $>6480$ & 66.0 & 23 & 0.0 & 6 & 11674 & $* 3947$ & 30.2 \\
\hline NaerumG2008 & 24104 & 118370 & - & 117894 & - & $>6480$ & - & 7 & 0.0 & 0 & 117894 & - & - \\
\hline \multirow[t]{2}{*}{ NaerumG2009 } & 7667 & 100450 & - & 6681 & $* 5114$ & $>6480$ & 0.3 & $>720$ & 6.2 & 0 & 5466 & 5113 & 6.4 \\
\hline & & & & & & & & & & & \multicolumn{3}{|c|}{ Continued on next page } \\
\hline
\end{tabular}


Table 1 - continued from previous page

Previous methods $\mathrm{TSD}^{\text {RoomLB }}$

\begin{tabular}{|c|c|c|c|c|c|c|c|c|c|c|c|c|c|}
\hline \multirow[b]{3}{*}{ Dataset } & \multirow{3}{*}{$\frac{\text { ALNS }}{\text { Obj }}$} & \multirow{2}{*}{\multicolumn{2}{|c|}{ 3-index model }} & \multirow{2}{*}{\multicolumn{2}{|c|}{ TSD }} & \multirow{2}{*}{\multicolumn{2}{|c|}{ Stage I }} & \multirow{2}{*}{\multicolumn{3}{|c|}{ Stage II }} & & & \\
\hline & & & & & & & & & & & \multirow[b]{2}{*}{ Obj } & \multirow[b]{2}{*}{ LB } & \multirow[b]{2}{*}{$\overline{\text { Gap }}$} \\
\hline & & Obj & LB & Obj & LB & Time & Gap & Time & Gap & Diff. & & & \\
\hline NielsSG2011 & 4953 & 10464 & 3323 & 6132 & *3412 & $>6480$ & 37.6 & 9 & 0.0 & 0 & 5397 & 3367 & 31.1 \\
\hline NielsSG2012 & 6952 & 12747 & 5722 & 8003 & *5738 & $>6480$ & 37.6 & 14 & 0.0 & 4 & 9192 & 5724 & 17.5 \\
\hline NordfynG2012 & 5160 & 8201 & $* 4152$ & 4890 & 4048 & $>6480$ & 23.3 & 35 & 0.0 & 59 & 5510 & 4107 & 15.1 \\
\hline NyborgG2011 & 13944 & 94059 & - & 31809 & $* 6129$ & $>6480$ & - & 7 & 0.0 & 4 & 85816 & - & 56.0 \\
\hline OdderCfU2010 & 18219 & 59540 & - & 40032 & 12188 & $>6480$ & 66.9 & 66 & 0.0 & 2 & 38875 & $* 12865$ & 29.4 \\
\hline OdderG2009 & 9308 & 59851 & - & 57586 & - & $>6480$ & 78.1 & 67 & 0.0 & 67 & 24686 & $* 5361$ & 42.4 \\
\hline OdderG2012 & 12307 & 17402 & 9602 & 14888 & 8878 & $>6480$ & 64.2 & 4 & 0.0 & 57 & 27199 & *9688 & 21.3 \\
\hline OrdrupG2010 & 13663 & 75700 & - & 12936 & 10665 & $>6480$ & 39.5 & 10 & 0.0 & 0 & 18101 & $* 10810$ & 16.4 \\
\hline OrdrupG2011 & 21612 & 116400 & - & 31329 & 16904 & $>6480$ & 38.7 & 305 & 0.0 & 8 & 28884 & $* 17692$ & 18.1 \\
\hline RibeK2011 & 21679 & 61945 & - & 43175 & 16209 & $>6480$ & 53.8 & 229 & 0.0 & 5 & 39107 & $* 18055$ & 16.7 \\
\hline RysenG2010 & 39971 & 110690 & - & 110690 & - & $>6480$ & - & 6 & 0.0 & 0 & 110690 & - & - \\
\hline RysenG2011 & 22260 & 100313 & - & 25989 & 17756 & $>6480$ & 71.4 & 9 & 0.0 & 5 & 68927 & *19725 & 11.4 \\
\hline RysenG2012 & 19841 & 110111 & - & 22156 & 15115 & $>6480$ & 71.7 & 14 & 0.0 & 15 & 59124 & $* 16708$ & 15.8 \\
\hline SanktAG2012 & 4207 & 4624 & 3415 & 3911 & 3376 & $>6480$ & 0.7 & $>720$ & 0.5 & 39 & 3721 & *3538 & 4.9 \\
\hline SkanderG2010 & 7209 & 7708 & 6051 & 6875 & 5712 & $>6480$ & 0.6 & $>720$ & 0.5 & 72 & 6485 & $* 6238$ & 3.8 \\
\hline SkanderG2011 & 22525 & 88470 & - & 88470 & - & $>6480$ & - & 5 & 0.0 & 0 & 88470 & - & - \\
\hline SkanderG2012 & 20138 & 98487 & - & 95319 & - & $>6480$ & - & 7 & 0.0 & 3 & 95319 & - & - \\
\hline SkiveG2010 & 43120 & 194740 & - & 194740 & - & $>6480$ & - & 526 & 0.0 & 0 & 194740 & - & - \\
\hline SlagelG2012 ${ }^{\dagger}$ & 32167 & 162960 & - & 162765 & - & $>6480$ & - & 417 & 0.0 & 0 & 162960 & - & - \\
\hline SoendS2011 & 11776 & 83560 & - & 83560 & - & $>6480$ & - & 131 & 0.0 & 0 & 83560 & - & - \\
\hline SoendS2012 & 8420 & 17778 & *6838 & 11915 & 6647 & $>6480$ & 72.5 & 8 & 0.0 & 4 & 24668 & 6739 & 18.8 \\
\hline StruerS2012 & 73361 & - & - & 207488 & - & $>6480$ & - & 700 & 0.0 & 0 & 211960 & - & - \\
\hline VardeG2012 & 10777 & 20933 & *5921 & 20622 & 5720 & $>6480$ & 72.1 & 12 & 0.0 & 2 & 20496 & 5668 & 45.1 \\
\hline VejenG2009 & 11264 & 69450 & - & 69450 & - & $>6480$ & 73.9 & $>720$ & 0.0 & 7 & 27954 & $* 7290$ & 35.3 \\
\hline Vejlefjo2011 & 13514 & 52035 & - & 18043 & 8511 & $>6480$ & 60.0 & 456 & 0.0 & 3 & 22066 & $* 8805$ & 34.8 \\
\hline VestfynG2009 & 5973 & 11606 & 4176 & 5999 & 4137 & $>6480$ & 14.5 & 553 & 0.0 & 18 & 5032 & $* 4211$ & 16.3 \\
\hline VestfynG2010 & 6761 & 16895 & $* 4308$ & 5974 & 4225 & $>6480$ & 16.3 & 65 & 0.0 & 21 & 5239 & 4290 & 17.8 \\
\hline VestfynG2011 & 7013 & 13624 & 5110 & 6657 & 4925 & $>6480$ & 19.6 & 38 & 0.0 & 24 & 6522 & $* 5159$ & 20.9 \\
\hline VestfynG2012 & 5244 & 11095 & 4279 & 5212 & 4210 & $>6480$ & 17.5 & 149 & 0.0 & 21 & 5319 & $* 4315$ & 17.2 \\
\hline ViborgK2011 & 14923 & 99170 & - & 99170 & - & $>6480$ & - & 6 & 0.0 & 0 & 99170 & - & - \\
\hline ViborgTG2009 & 10216 & 19891 & 8695 & 12077 & 8356 & $>6480$ & 34.7 & 45 & 0.0 & 3 & 13387 & $* 8740$ & 14.4 \\
\hline ViborgTG2010 & 4932 & 12727 & 4130 & 10226 & 3990 & $>6480$ & 60.9 & 11 & 0.0 & 19 & 10665 & $* 4146$ & 15.9 \\
\hline ViborgTG2011 & 7478 & 16433 & 6716 & 9808 & 6204 & $>6480$ & 38.8 & 12 & 0.0 & 13 & 11088 & *6772 & 9.4 \\
\hline VirumG2012 & 27738 & 140883 & - & 32183 & 17770 & $>6480$ & 75.3 & 16 & 0.0 & 9 & 79111 & *19486 & 29.7 \\
\hline VordingbG2009 & 8568 & 17025 & 5457 & 9905 & 5243 & $>6480$ & 33.6 & 10 & 0.0 & 167 & 8972 & $* 5787$ & 32.5 \\
\hline Avg. & & & & & & & 44.3 & & 0.1 & 17.9 & & & 22.3 \\
\hline
\end{tabular}

$\dagger$ An artificial bound on the amount of Halls' inequalities generated was enforced for tractability, see Section 4.3.

Table 2: Results summary. Note that rows 'Best solution' and 'Best bound' also counts draws.

\begin{tabular}{lcccc} 
& ALNS & 3-index model & TSD & TSD $^{\text {RoomLB }}$ \\
\hline Solution found & 100 & 99 & 100 & 100 \\
Best solution & 77 & 2 & 8 & 18 \\
Bound found & - & 46 & 79 & 80 \\
Best bound & - & 13 & 19 & 49
\end{tabular}

Table 3: Comparison of the amount of best found solutions for the exact methods. Draws are also counted.

\begin{tabular}{cccc} 
& 3-index model & TSD & TSD $^{\text {RoomLB }}$ \\
\hline Best solution & 16 & 66 & 52
\end{tabular}

A number of conclusions can be drawn from the numbers: 
- For 97 instances, the solution obtained by $\mathrm{TSD}^{\mathrm{RoomLB}}$ is at least as good as the solution obtained by the 3-index model, and for most instances significantly better.

- $\mathrm{TSD}^{\mathrm{RoomLB}}$ is generally the best method for generating bounds, finding the best bound on 49 instances overall.

- $\mathrm{TSD}^{\mathrm{RoomLB}}$ was capable of finding a lower bound for 80 instances. This means that for 20 instances, Gurobi was unable to solve the LP-relaxation of the root node of the Stage I model within the timelimit. Table 4 shows statistics for these instances (the presolved models). It is seen that the problems does not contain coefficients of huge magnitude in neither the objective, system matrix, or rhs. Hence the issue seems related to the relatively big number of constraints and variables. In average, these instances have more than 100000 constraints and variables, hence we think its fair to consider them as large-scale. Note that if the root-LP was not solved for an instance, the reported solution replicates the initial solution provided by us to Gurobi (Gurobi apparently starts its solution process by verifying the feasibility of the MIP Start attributes).

- $\mathrm{TSD}^{\text {RoomLB }}$ produces the best solution for 18 instances overall, while TSD produces the best solution on 8 instances. The ALNS heuristic is best on 77 instances, and is currently the best algorithm for this problem (keep in mind the ALNS algorithm was allowed significantly less CPU time).

- Comparing the exact methods (Table 3), it is seen that it is generally not profitable to use the extended Stage I model if the goal is to obtain good solutions. Both variants of the decomposition finds more best solutions than the pure 3-index model.

- The Stage I model of TSD ${ }^{\text {RoomLB }}$ is a challenge for Gurobi, with an average gap of $44.3 \%$ over all instances where a LB was found. Only 4 instances are solved to optimality, and these are among the smallest instances (see Sørensen and Stidsen (2013) for instance statistics).

- The Stage II model of TSD ${ }^{\text {RoomLB }}$ is in general easy to solve. The average gap for this model over all problem instances is $0.1 \%$, and 89 instances are solved to optimality. This means that future research can focus on solving the Stage I model.

- The difference between the lower bound on room allocation and the actual allocation of rooms (column 'Diff.') is low, compared to the magnitude of objectives in general. This means that only a small increase in solution quality can be gained by improving the bound on room allocation.

Table 4: Statistics of the Stage I models (after presolve) where Gurobi was unable to solve the LP-relaxation of the root-node within the timelimit (i.e. for those instances where the TSD ${ }^{\text {RoomLB }}$ was unable to provide a lower bound). Column 'Cons.' shows the number of constraints and 'Non-zeros' shows the amount of non-zeros in the model. 'Variables' shows the amount of continuous, integer and binary variables. 'Obj. coef.', 'Model coef.', and 'RHS coef' shows the smallest and largest coefficient in the objective function, system matrix and right-hand side, respectively.

\begin{tabular}{|c|c|c|c|c|c|c|c|c|c|c|c|}
\hline & \multirow[b]{2}{*}{ Cons. } & \multirow[b]{2}{*}{ Non-zeros } & \multicolumn{3}{|c|}{ Variables } & \multicolumn{2}{|c|}{ Obj. coef. } & \multicolumn{2}{|c|}{ Model coef. } & \multicolumn{2}{|c|}{ RHS coef. } \\
\hline & & & Cont. & Integer & Binary & Min. & Max. & Min. & Max. & Min. & Max. \\
\hline Min. & 73293 & 881433 & 240 & 525 & 4 & 1 & 84 & 1 & 4 & 0 & 6 \\
\hline Max. & 167978 & 4200206 & 52015 & 252514 & 246874 & 1 & 1120 & 1 & 16 & 1 & 103 \\
\hline Avg. & 118396 & 1967455 & 36918 & 101460 & 88321 & 1 & 264 & 1 & 7 & 1 & 32 \\
\hline
\end{tabular}

As an extension to the decomposition, one could use the ALNS heuristic to provide a starting solution. Since the ALNS heuristic is able to produce a fairly good solution quickly, this would most likely lead to improved performance.

As a loose remark, we mention that Burke et al. (2010) formulates an IP of the Udine Course Timetabling Problem (used in the International Timetabling Competition 2007), using a three-indexed binary variable, and reports that CPLEX 11 uses up to 6400 seconds when solving the root LP (using Dual Simplex, which is also used by Gurobi as default). Their model is quite similar in structure to ours, so possibly this class of IP formulations contain undesirable properties in the eyes of general-purpose MIP solvers. 


\section{Conclusion}

A Two-Stage Decomposition for a real-world high school timetabling problem has been shown. This splits the Integer Programming model into two smaller models, which reduces the number of variables significantly. Computational results show that this approach is way more effective than solving the usual original IP with a 3-index binary variable, in terms of both the obtained solutions and the obtained bounds. This constitutes the TSD as the best exact method for solving this particular timetabling problem. However, the integration of the lower bound on room allocation in the Stage I model has bad influence on the quality of solutions, but makes the decomposition capable of achieving better lower bounds. Nevertheless this extension of the Stage I model represents interesting theory which can likely be used in the context of decomposing other timetabling problems.

For other types of (timetabling) problems, this type of decomposition might be a way of enhancing computational times. However, a special structure is required for applying the decomposition, which limits the set of applicable problems. On the other hand, the advantage gained by reducing the number of variables should not be underestimated, and we encourage researchers to attempt this type of decomposition if possible.

Approximating the room allocation penalties in the Stage I model is an interesting approach, and also sets a possible agenda for future work; 1) Can a better approximation (or even the exact value) be found for the minimum weight maximum matching problem representing room penalties? 2) Can the room stability penalties be incorporated in the Stage I model? However, the most important issue for future research is a more efficient way of solving the Stage I model. This is the bottleneck of the TSD for this particular problem.

\section{References}

Avella, P., D’Auria, B., Salerno, S., Vasilâev, I., 2007. A computational study of local search algorithms for italian high-school timetabling. Journal of Heuristics 13, 543-556.

Badri, M. A., 1996. A two-stage multiobjective scheduling model for [faculty-course-time] assignments. European Journal of Operational Research 94 (1), $16-28$.

Balas, E., Pulleyblank, W., 1983. The perfectly matchable subgraph polytope of a bipartite graph. Networks 13 (4), $495-516$.

Bardadym, V., 1996. Computer-aided school and university timetabling: The new wave. In: Burke, E., Ross, P. (Eds.), Practice and Theory of Automated Timetabling. Vol. 1153 of Lecture Notes in Computer Science. Springer Berlin / Heidelberg, pp. $22-45$.

Birbas, T., Daskalaki, S., Housos, E., 1997. Timetabling for greek high schools. Journal of the Operational Research Society 48, 1191-1200(10).

Birbas, T., Daskalaki, S., Housos, E., April 2009. School timetabling for quality student and teacher schedules. J. of Scheduling $12,177-197$.

Burke, E., Marecek, J., Parkes, A., Rudová, H., 2010. Decomposition, reformulation, and diving in university course timetabling. Computers \& Operations Research 37 (3), 582-597.

Burke, E., Newall, J. P., 1999. A multistage evolutionary algorithm for the timetable problem. Evolutionary Computation, IEEE Transactions on $3(1), 63-74$.

Carter, M., 1983. A decomposition algorithm for practical timetabling problems. Tech. Rep. 83-06, Department of Industrial Engineering, University of Toronto.

Daskalaki, S., Birbas, T., 2005. Efficient solutions for a university timetabling problem through integer programming. European Journal of Operational Research 160 (1), $106-120$

Daskalaki, S., Birbas, T., Housos, E., 2004. An integer programming formulation for a case study in university timetabling. European Journal of Operational Research 153, 117-135.

de Werra, D., 1985. An introduction to timetabling. European Journal of Operational Research 19 (2), 151 - 162.

Dimopoulou, M., Miliotis, P., 2001. Implementation of a university course and examination timetabling system. European Journal of Operational Research 130 (1), $202-213$.

Edmonds, J., 1965. Maximum matching and a polyhedron with 0, 1-vertices. Journal of Research of the National Bureau of Standards B 69, $125-130$.

Egerváry, E., 1931. Matrixok kombinatorius tulajdonságairól. Matematikai és Fizikai Lapok 38, 16-28.

Gotlieb, C. C., 1962. The construction of class-teacher timetables. In: Popplewell, C. M. (Ed.), IFIP Congress. Vol. 62. North-Holland Pub. Co, pp. 73-77.

Lach, G., Lübbecke, M., 2008. Optimal university course timetables and the partial transversal polytope. In: McGeoch, C. (Ed.), Experimental Algorithms. Vol. 5038 of Lecture Notes in Computer Science. Springer Berlin / Heidelberg, pp. 235-248.

Lach, G., Lübbecke, M., 2012. Curriculum based course timetabling: new solutions to udine benchmark instances. Annals of Operations Research 194, 255-272.

Lawrie, N. L., 1969. An integer linear programming model of a school timetabling problem. The Computer Journal 12 (4), $307-316$.

Lovász, L., Plummer, M. D., 2009. Matching Theory. AMS Chelsea Publishing.

MirHassani, S., 2006. A computational approach to enhancing course timetabling with integer programming. Applied Mathematics and Computation $175(1), 814-822$.

Mittelman, H., Aug. 2013. Benchmarks for optimization software. http://plato.asu.edu/bench.html [Retrieved 20/8-2013]. 
Papoutsis, K., Valouxis, C., Housos, E., 2003. A column generation approach for the timetabling problem of greek high schools. The Journal of the Operational Research Society 54 (3), 230-238.

Pillay, N., February 2013. A survey of school timetabling research. Annals of Operations Research.

Post, G., Ahmadi, S., Daskalaki, S., Kingston, J., Kyngas, J., Nurmi, C., Ranson, D., 2012a. An xml format for benchmarks in high school timetabling. Annals of Operations Research 194, 385-397.

Post, G., Gaspero, L. D., Kingston, J. H., McCollum, B., Schaerf, A., August 2012b. The third international timetabling competition. In: Proceedings of the Ninth International Conference on the Practice and Theory of Automated Timetabling (PATAT 2012). Son, Norway.

Qualizza, A., Serafini, P., 2005. A column generation scheme for faculty timetabling. In: Burke, E., Trick, M. (Eds.), Practice and Theory of Automated Timetabling V. Vol. 3616 of Lecture Notes in Computer Science. Springer Berlin Heidelberg, pp. 161-173.

Santos, H., Uchoa, E., Ochi, L., Maculan, N., April 2012. Strong bounds with cut and column generation for class-teacher timetabling. Annals of Operations Research 194 (1), 399-412.

Schaerf, A., 1999. A survey of automated timetabling. Artificial Intelligence Review 13, 87-127.

Schrijver, A., 2003. Combinatorial optimization: polyhedra and efficiency. Vol. 24 of Algorithms and Combinatorics. Springer

Sørensen, M., Stidsen, T., March 2013. Comparing solution approaches for a complete model of high school timetabling. Tech. Rep. 5.2013, DTU Management Engineering, Technical University of Denmark. 